\title{
Online Identification of Multivariable Discrete Time Delay Systems Using a Recursive Least Square Algorithm
}

\author{
Saïda Bedoui, Majda Ltaïef, and Kamel Abderrahim \\ National Engineering school of Gabes, Numerical Control of Industrial Processes Research Unit, Gabes University, \\ Route de Medenine, BP 6029, Gabes, Tunisia \\ Correspondence should be addressed to Saïda Bedoui; saida.bedoui@enig.rnu.tn
}

Received 6 February 2013; Revised 30 April 2013; Accepted 25 May 2013

Academic Editor: Yang Yi

Copyright (C) 2013 Saïda Bedoui et al. This is an open access article distributed under the Creative Commons Attribution License, which permits unrestricted use, distribution, and reproduction in any medium, provided the original work is properly cited.

\begin{abstract}
This paper addresses the problem of simultaneous identification of linear discrete time delay multivariable systems. This problem involves both the estimation of the time delays and the dynamic parameters matrices. In fact, we suggest a new formulation of this problem allowing defining the time delay and the dynamic parameters in the same estimated vector and building the corresponding observation vector. Then, we use this formulation to propose a new method to identify the time delays and the parameters of these systems using the least square approach. Convergence conditions and statistics properties of the proposed method are also developed. Simulation results are presented to illustrate the performance of the proposed method. An application of the developed approach to compact disc player arm is also suggested in order to validate simulation results.
\end{abstract}

\section{Introduction}

Time delay system identification has received great attention in the last years since time delay is a physical phenomenon which arises in most control loops industrial systems $[1,2]$. Several reasons cause the presence of time delay in control loops. In fact, it may be an inherent feature of the system such as processes of transport (mass, energy, and information), higher order processes, and accumulation of time lags in several systems that are connected in series. It may also be introduced by the devices of control loops, such as response times of sensors and actuators, computation time of control laws, and information transmission time in networks. This delay can be neglected if its value is too small for the system time constants. Otherwise, it cannot be neglected, and the dynamic representation of the system must be described by a time delay model. This model is, generally, constructed using the identification approach which allows building a mathematical model from input-output data.

The identification of time delay systems is known to be a challenging identification problem because it involves both the estimation of dynamic parameters and time delay. Numerous methods have been proposed in the literature for the identification of time delay systems [3-15].
Among these methods, the graphical approach has been the most popular since it represents the first method proposed in the literature for the identification of continuous time delay systems [16]. Moreover, it is frequently used to compute the parameters of PID controllers for industrial processes. It consists in determining the time delay and the dynamic parameters of the system from its step response. The main advantage of this approach lies in the simplicity of its implementation. However, it produces inaccurate results because it is very sensitive to measurement noises. Another popular approach is proposed in [9]. It is based on the approximation of the time delay by a rational transfer function using classical approximations such as Pade or Laguerre. This method can insure very satisfactory results in the case of linear systems with constant time delays and lower order. However, its performance degrades rapidly in the case of higher order systems or important time delays. Moreover, it raises the computational complexity because it increases the number of parameters to be estimated. The parametrisation approach can be considered as one of the interesting methods because it is based on theoretical concepts of discrete time systems [17]. It consists, firstly, in inserting a known time delay in the numerator of the discrete time model, secondly, in estimating the dynamic 
parameters of the system using a recursive algorithm, and, finally, in deducing the time delay from zero coefficients of the numerator. In practice, it is difficult or rather impossible to have zero coefficients from experimental data. Indeed, we must set a threshold, which is a delicate task, mainly in the case of a noisy output. The method developed in [3] consists, It consists, firstly, in using the recursive least square approach to identify the parameters assuming that the time delay is known, and secondly, in estimating the time delay, taking into account the results of the first step. The time delay may be identified either by maximizing the correlation function or by minimizing the quadratic error. This method assumes that the domain range of the time delay is a priori known. We also mention the method presented in [18]. It allows the identification of the time delay and the system parameters using the Levenberg-Marquaydt optimization approach to minimize the prediction error. An online identification algorithm for continuous-time singleinput single-output (SISO) linear time delay systems with uncertain time invariant parameters is developed in [10]. It consists in constructing a sliding mode-based observer of an underlying system with uncertain parameters. This observer is then used to design an adaptive identifier of system parameters. A linear filtering method is introduced in [19] for simultaneous parameter and time delay estimation of transfer function models. This method estimates the time delay along other model using an iterative way through simple linear regression. Another method that identifies the time delay and the system parameters is presented in [20] which is based on the correlation technique. The method developed in [21] allows the identification of time delay and the parameters of a system operating in the presence of colored noise. This method is based on correlation analysis. The method developed in $[12,22]$ allows the identification of time delay and the parameters. It minimizes the error between the process output and the process predictive model output, and then the variable time delay parameter is identified. In our previous work, we have proposed two methods for the simultaneous identification of the time delay and dynamic parameters of monovariable time delay discrete systems. The first method is based on the least square approach [23, 24]. The second method consists in minimizing a quadratic criterion using the gradient approach [25].

Most of these approaches deal with the problem of the identification of single-input single-output (SISO) time delay systems. However, the problem of multi-input multioutput (MIMO) time delay systems is one of the most difficult problems that represents an area of research where few efforts have been devoted in the past. The use of time delay approximation is extended to the MIMO case [26]. In fact, the authors deal with the problem of the identification of time delay processes using an overparameterization method. In [27], a method is developed for time delay estimation in the frequency domain of MIMO systems based on the combination of continuous wavelet transform (CWT) and cross-correlation. During the estimation, cross-correlation computations are carried out between the CWT coefficients of the input and the output data. The authors of [28] have proposed a simple method based on the combination of two well-known approaches: time delay estimation from impulse response and subspace identification.

In this paper, we propose an alternative approach for the problem of simultaneous identification of linear discrete time delay multivariable systems. Indeed, we develop a new formulation of the problem allowing to define the time delay and the dynamic parameters in the same estimated vector and to build the corresponding observation vector. Then, we use this formulation to propose a new method to identify the time delays and the parameters of these systems using the least square approach. Convergence conditions and statistics properties of the proposed method are also developed. Simulation and experimental examples are presented to illustrate the effectiveness of the proposed methods and to compare their performance in terms of convergence and speed. Our approach presents several interesting properties which can be summarized as follows.

(i) The simultaneous identification of the time delays and parameters matrices is achieved by a new formulation of the parameters matrices.

(ii) No a priori knowledge of the time delay is required. In fact, most of the publications assume the knowledge of the time delay variation range or the initial condition.

(iii) The consistency of recursive least square methods has received much attention in the identification literature. In this paper, the proof of the consistency of the estimates is established.

(iv) It can be used to deal with control adaptive purposes.

This paper is organized as follows. Section 2 presents the model and its assumptions. In Section 3, we propose an extended least square algorithm for simultaneous online identification of unknown time delays and parameters of multivariable discrete time delay systems. Moreover, we develop the convergence properties of the estimates in order to show that the obtained estimates are unbiased. Simulation results and experimental test are provided in the last section.

\section{Problem Statement}

In this paper, we address the problem of identification of square linear multivariable delay system with $p$ inputs and p ARX model:

$$
A\left(q^{-1}\right) Y(k)=B\left(q^{-1}\right) \underline{U}(k)+V(k)
$$

where $Y(k)=\left[y_{1}(k), \ldots, y_{p}(k)\right]^{T}$ and $\underline{U}(k)=\left[U_{1}(k-\right.$ $\left.\left.d_{1}\right), \ldots, U_{p}\left(k-d_{p}\right)\right]^{T}$ are the outputs and the delayed inputs of the system at time $k$ and $V(k)=\left[V_{1}(k), \ldots, V_{p}(k)\right]^{T}$ is a vector of independent random variables sequences. Let $\{D=$ $\left.\operatorname{diag}\left[d_{1}, \ldots, d_{p}\right] / d_{i} \in \mathbb{N}, i=1, \ldots, p\right\}$ be the time delay diagonal matrix, also called the interactive matrix, and $A\left(q^{-1}\right)$ and $B\left(q^{-1}\right)$ two polynomial matrices in the unit backward 
shift operator $q^{-1}$ (i.e., $\left.q^{-1} y_{i}(k)=y_{i}(k-1), i=1, \ldots, p\right)$, defined by

$$
\begin{gathered}
A\left(q^{-1}\right)=I_{p}+A_{1} q^{-1}+\cdots+A_{n_{a}} q^{-n_{a}}, \\
\operatorname{dim} A_{r}=(p, p), \quad r \in\left[1, n_{a}\right], \\
B\left(q^{-1}\right)=B_{1} q^{-1}+\cdots+B_{n_{b}} q^{-n_{b}}, \\
\operatorname{dim} B_{r}=(p, p), \quad r \in\left[1, n_{b}\right] .
\end{gathered}
$$

The delayed inputs $\underset{U}{U}(k)$ can be expressed as

$$
\underline{U}(k)=\Omega U(k),
$$

where $\Omega$ is a diagonal matrix defined as

$$
\Omega=\left(\begin{array}{cccc}
q^{-d_{1}} & 0 & \cdots & 0 \\
0 & q^{-d_{2}} & \ddots & \vdots \\
\vdots & \ddots & \ddots & 0 \\
0 & \cdots & 0 & q^{-d_{p}}
\end{array}\right) .
$$

The following assumptions are made.

(A1) The two polynomial matrices $A\left(q^{-1}\right)$ and $B\left(q^{-1}\right)$ have no common left factor.

(A2) The orders $n_{a}$ and $n_{b}$ of the model are known.

(A3) The input sequences $\left\{\underline{U}=\left[U_{1}\left(k-d_{1}\right), \ldots, U_{p}(k-\right.\right.$ $\left.\left.\left.d_{p}\right)\right]^{T}\right\}$ are independent of $V(k)$, mutually independent and identically distributed with $E[U(k)]=0$ and $E\left[U(k) U(k)^{T}\right]=I$, and are persistently exciting $(\mathrm{PE})$.

(A4) The disturbance $V(k)=\left[V_{1}(k), \ldots, V_{p}(k)\right]^{T}$ is sequences of independent, identically distributed random variables with zero mean and finite variance $\Sigma=$ $\left\{\sigma_{1}^{2}, \ldots, \sigma_{p}^{2}\right\}$.

(A5) The inputs, the outputs, and the noises are causal; that is, $U(k)=[0], Y(k)=[0]$, and $V(k)=[0]$ for $k \leqslant 0$.

Problem Statement. The goal is to develop a recursive algorithm to estimate, simultaneously, the time delay matrix $D$ and the matrices $\left\{A_{i}(k), B_{i}(k)\right\}$ using the input/output measurement data $\{U(k), Y(k)\}$.

In the following, we present three necessary definitions.

Definition 1. Operator round $(d)$ is defined by

$$
\text { round }\left(d_{i}\right)= \begin{cases}\operatorname{int}\left(d_{i}\right)+1 & \text { if } d_{i}-\operatorname{int}\left(d_{i}\right) \geqslant 0.5 \\ \operatorname{int}\left(d_{i}\right) & \text { if } d_{i}-\operatorname{int}\left(d_{i}\right)<0.5,\end{cases}
$$

where int $(d)$ denotes the integer part of $d_{i}, i=1, \ldots, p$.

Definition 2. Operator $\widetilde{d}(\cdot)$ is defined by

$$
\widetilde{d}_{i}(\cdot)=\operatorname{round}\left(\widehat{d}_{i}(\cdot)\right), \quad i=1, \ldots, p .
$$

Definition 3. Operator $\widetilde{D}(\cdot)$ is defined by

$$
\widetilde{D}(\cdot)=\left(\begin{array}{cccc}
\tilde{d}_{1} & 0 & \cdots & 0 \\
0 & \widetilde{d}_{2} & \ddots & \vdots \\
\vdots & \ddots & \ddots & 0 \\
0 & \cdots & 0 & \tilde{d}_{p}
\end{array}\right) .
$$

\section{The Proposed Approach}

In this section, an extended least square algorithm for simultaneous online identification of time delays and parameter matrices is developed.

Equation (1) can be rewritten as

$$
Y(k)=\Theta^{T} \varphi(k, D)+V(k),
$$

where $\Theta$ is the parameter matrix and $\varphi(k, D)$ is the observation vector defined as

$$
\begin{gathered}
\Theta^{T}=\left[A_{1}, A_{2}, \ldots, A_{n_{a}}, B_{1}, B_{2}, \ldots, B_{n_{b}}\right], \\
\varphi(k, D)=\left[-Y^{T}(k-1),-Y^{T}(k-2), \ldots,-Y^{T}\left(k-n_{a}\right),\right. \\
\left.\underline{U}^{T}(k-1), \ldots, \underline{U}^{T}\left(k-n_{b}\right)\right]^{T} .
\end{gathered}
$$

On the other hand, the estimated output is described by the following relation:

$$
\widehat{Y}(k)=\widehat{\Theta}^{T} \varphi(k, \widetilde{D}),
$$

where $\widehat{\Theta}$ and $\widetilde{D}=\operatorname{diag}\left[\widetilde{d}_{1}, \ldots, \widetilde{d}_{p}\right]$ represent, respectively, the estimated parameter matrix and the estimated time delay matrix.

Let us consider the prediction error $\Upsilon(k)$ given by

$$
\Upsilon(k)=Y(k)-\widehat{\Theta}^{T} \varphi(k, \widetilde{D}) .
$$

Since parameter matrix $\widehat{\Theta}$ does not contain the unknown time delays $\widetilde{D}$, then consequently it is not directly applicable to achieve our objective which is the simultaneous identification of the time delays and the parameter matrices of the multivariable discrete time delay systems (1).

To overcome this problem, we suggest considering the time delay matrix in parameter matrices $\Theta$ to be estimated. Indeed, the new matrix, called generalized matrix, is given by

$$
\Theta_{G}^{T}=[\Theta, D] .
$$

Moreover, we propose the use of the negative gradient of the error to obtain an appropriate observation vector which is given by

$$
\Phi\left(k, \widehat{\Theta}_{G}\right)=-\frac{\partial \Upsilon}{\partial \widehat{\Theta}_{G}} .
$$


Then,

$$
\begin{gathered}
\Phi\left(k, \widehat{\Theta}_{G}\right)=\left[\varphi^{T}(k, \widetilde{D}),-\frac{\partial \Upsilon}{\partial \widetilde{D}}\right]^{T}, \\
\Phi\left(k, \widehat{\Theta}_{G}\right)=\left[\varphi^{T}(k, \widetilde{D}), \frac{\partial \widehat{Y}}{\partial \widetilde{D}}\right]^{T}, \\
\Phi\left(k, \widehat{\Theta}_{G}\right)=\left[\varphi^{T}(k, \widetilde{D}), \frac{\partial}{\partial \widehat{D}} \sum_{i=1}^{n_{b}} U^{T}(k-i) \widehat{B}, \widehat{\Omega}\right]^{T} .
\end{gathered}
$$

The use of the approximation of $\operatorname{Ln}(q) \approx 1-q^{-1}$ (see the appendix) leads to

$$
\Phi\left(k, \widehat{\Theta}_{G}\right)=\left[\varphi^{T}(k, \widetilde{D}),-\sum_{i=1}^{n_{b}} \Delta U^{T}(k-i) \widehat{B}_{i} \widehat{\Omega}\right]^{T},
$$

where $\Delta U(k)=U(k)-U(k-1)$.

Replacing $\varphi^{T}(k, \widetilde{D})$ by its expression, we obtain the generalized observation vector:

$$
\Phi\left(k, \widehat{\Theta}_{G}\right)=\left[\begin{array}{c}
-Y^{T}(k-1) \\
\vdots \\
-Y^{T}\left(k-n_{a}\right) \\
\widehat{\Omega} U^{T}(k-1) \\
\vdots \\
\widehat{\Omega} U^{T}\left(k-n_{b}\right) \\
-\sum_{i=1}^{n_{b}} \Delta U^{T}(k-i) \widehat{B}_{i} \widehat{\Omega}
\end{array}\right]
$$

An estimation $\widehat{\Theta}_{G}$ of $\Theta_{G}$ is denoted by the minimization of the following criterion:

$$
J\left(k, \Theta_{G}\right)=\frac{1}{2} \sum_{i=0}^{k} \Upsilon(i)^{2} .
$$

Then, the partial derivative of the criterion with respect to the generalized matrix is

$$
\frac{\partial J}{\partial \widehat{\Theta}_{G}}=\sum_{i=0}^{k} \frac{\partial \Upsilon(i)}{\partial \widehat{\Theta}_{G}} \Upsilon(i)=-\sum_{i=0}^{k} \Phi\left(i, \widehat{\Theta}_{G}\right) \Upsilon(i) .
$$

So

$$
\frac{\partial J}{\partial \widehat{\Theta}_{G}}=-\sum_{i=0}^{k} \Phi\left(i, \widehat{\Theta}_{G}\right)\left[Y(i)-\widehat{\Theta}^{T} \varphi(i, \widetilde{D})\right] .
$$

Let us consider

$$
\psi=-\sum_{j=1}^{n_{b}} \widetilde{D} \Delta U^{T}(i-j) \widehat{B}_{j} \widehat{\Omega} .
$$

Adding and subtracting from (19) the term $\psi$, given by (20), we have

$$
\begin{aligned}
\frac{\partial J}{\partial \widehat{\Theta}_{G}} & =-\sum_{i=0}^{k} \Phi\left(i, \widehat{\Theta}_{G}\right)\left[Y(i)-\Phi^{T}\left(i, \widehat{\Theta}_{G}\right) \widehat{\Theta}_{G}+\psi\right] \\
& =-\sum_{i=0}^{k} \Phi\left(i, \widehat{\Theta}_{G}\right)\left[Y(i)+\psi-\Phi^{T}\left(i, \widehat{\Theta}_{G}\right) \widehat{\Theta}_{G}\right] .
\end{aligned}
$$

Canceling the partial derivative of the criterion, we obtain

$$
\begin{aligned}
\widehat{\Theta}_{G}(k)= & {\left[\sum_{i=0}^{k} \Phi\left(i, \widehat{\Theta}_{G}\right) \Phi^{T}\left(i, \widehat{\Theta}_{G}\right)\right]^{-1} \sum_{i=0}^{k} \Phi\left(i, \widehat{\Theta}_{G}\right) } \\
& \times[Y(i)+\psi] .
\end{aligned}
$$

Let,

$$
R(k)=\sum_{i=0}^{k} \Phi\left(i, \widehat{\Theta}_{G}\right) \Phi^{T}\left(i, \widehat{\Theta}_{G}\right) .
$$

Based on assumption A3 which ensures that matrix $R(k)$ is invertible [29], (22) can be rewritten as

$$
\widehat{\Theta}_{G}(k)=R(k)^{-1} \sum_{i=0}^{k} \Phi\left(i, \widehat{\Theta}_{G}\right)[Y(i)+\psi] .
$$

It follows that

$$
\begin{aligned}
\widehat{\Theta}_{G}(k)=R(k)^{-1}( & \sum_{i=0}^{k-1} \Phi\left(i, \widehat{\Theta}_{G}\right)[Y(i)+\psi] \\
& \left.+\Phi\left(k, \widehat{\Theta}_{G}\right)[Y(k)+\psi]\right) .
\end{aligned}
$$

Using (22), we have

$$
\begin{aligned}
\widehat{\Theta}_{G}(k)=R(k)^{-1}( & R(k-1) \widehat{\Theta}_{G}(k-1) \\
& \left.+\Phi\left(k, \widehat{\Theta}_{G}\right)[Y(k)+\psi]\right) \\
=R(k)^{-1}( & R(k) \widehat{\Theta}_{G}(k-1) \\
& -\Phi\left(k, \widehat{\Theta}_{G}\right) \Phi^{T}\left(k, \widehat{\Theta}_{G}\right) \widehat{\Theta}_{G}(k-1) \\
& \left.+\Phi\left(k, \widehat{\Theta}_{G}\right)[Y(k)+\psi]\right) .
\end{aligned}
$$

So

$$
\begin{aligned}
\widehat{\Theta}_{G}(k)= & \widehat{\Theta}_{G}(k-1)+R(k)^{-1} \Phi\left(k, \widehat{\Theta}_{G}\right) \\
& \times\left(-\Phi^{T}\left(k, \widehat{\Theta}_{G}\right) \widehat{\Theta}_{G}(k-1)+[Y(k)+\psi]\right) .
\end{aligned}
$$

It follows from (27) that

$$
\begin{aligned}
\widehat{\Theta}_{G}(k)= & \widehat{\Theta}_{G}(k-1)+R(k)^{-1} \Phi\left(k, \widehat{\Theta}_{G}\right) \\
& \times\left(Y(k)-\widehat{\Theta}^{T} \varphi(k, \widetilde{D})\right) .
\end{aligned}
$$

Thus,

$$
\begin{aligned}
\widehat{\Theta}_{G}(k) & =\widehat{\Theta}_{G}(k-1)+P(k) \Phi\left(k, \widehat{\Theta}_{G}\right) \Upsilon(k), \\
P(k) & =\left[\sum_{i=0}^{k} \Phi\left(i, \widehat{\Theta}_{G}\right) \Phi^{T}\left(i, \widehat{\Theta}_{G}\right)\right]^{-1} \\
& =\left[R(k-1)+\Phi\left(k, \widehat{\Theta}_{G}\right) \Phi^{T}\left(k, \widehat{\Theta}_{G}\right)\right]^{-1} .
\end{aligned}
$$


Using the matrix inversion lemma given by [29],

$$
\left[B+C D^{T}\right]^{-1}=B^{-1}-B^{-1} C D^{T} B^{-1}\left[1+D^{T} B^{-1} C\right]^{-1} .
$$

Let $B=R(k-1), C=\Phi\left(k, \Theta_{G}\right)$, and $D=\Phi\left(k, \Theta_{G}\right)^{T}$, and then we have

$$
P(k)=P(k-1)-\frac{P(k-1) \Phi\left(k, \widehat{\Theta}_{G}\right) \Phi^{T}\left(k, \widehat{\Theta}_{G}\right) P(k-1)}{1+\Phi^{T}\left(k, \widehat{\Theta}_{G}\right) P(k-1) \Phi\left(k, \widehat{\Theta}_{G}\right)} .
$$

The previous approach can be summarized by Algorithm 1 .

\section{Convergence Properties}

4.1. Consistency. For the system in (1), assume that (A3) and (A4) hold. Then for any $c>1$, the parameter estimation error, $\widehat{\Theta}_{G} \rightarrow \Theta_{G}$, associated with the LS algorithm in (29) and (30) satisfies

$$
\left\|\widehat{\Theta}_{G}-\Theta_{G}\right\|^{2}=O\left(\frac{[\operatorname{Ln} r(k)]^{c}}{\lambda_{\min }\left[P^{-1}(k)\right]}\right),
$$

where $r(k)$ is the trace of the covariance matrix $P^{-1}(k)$ and $\lambda_{\text {min }}$ represents the minimum eigenvalues of $P^{-1}(k)$.

Proof. Define the parameter estimation error vector:

$$
\bar{\Theta}_{G}:=\widehat{\Theta}_{G}-\Theta_{G} .
$$

Using (27) and (11) we have

$$
\begin{aligned}
\bar{\Theta}_{G}(k)= & \bar{\Theta}_{G}(k-1)+P \Phi\left(k, \Theta_{G}\right) \\
& \times\left[\widehat{\Theta}^{T}(k-1) \varphi(k, \widehat{D})\right. \\
& \left.\quad+V(k)-\Theta^{T}(k-1) \varphi(k, D)\right] \\
= & \bar{\Theta}_{G}(k-1)+P \Phi\left(k, \Theta_{G}\right)[-\bar{\rho}(k)+V(k)],
\end{aligned}
$$

where

$$
\bar{\rho}(k)=\widehat{\Theta}^{T}(k-1) \varphi(k, \widehat{D})-\Theta^{T}(k-1) \varphi(k, D) .
$$

Equation (35) can be rewritten as (see the appendix)

$$
\bar{\Theta}_{G}(k)=\bar{\Theta}_{G}(k-1)+P \Phi\left(k, \Theta_{G}\right)[-\bar{Y}(k)-\xi(k)+V(k)],
$$

where

$$
\begin{aligned}
\xi(k)= & \sum_{i=1}^{n b} B_{i}(\widehat{\Omega}-\Omega) U^{T}(k-i) \\
& +\sum_{i=1}^{n b}\left(\widehat{D} \widehat{B}_{i}-D B_{i}\right) \widehat{\Omega} \Delta U^{T}(k-i), \\
\bar{Y}(k)= & \bar{\Theta}_{G}^{T}(k-1) \Phi\left(k, \widehat{\Theta}_{G}\right) .
\end{aligned}
$$

Let us define now a nonnegative definite function:

$$
S(k):=\bar{\Theta}_{G}^{T}(k) P^{-1}(k) \bar{\Theta}_{G}(k) .
$$

Replacing $\bar{\Theta}_{G}^{T}(k)$ by its expression, we obtain

$$
\begin{aligned}
S(k)=[ & \bar{\Theta}_{G}(k-1)+P(k) \Phi\left(k, \widehat{\Theta}_{G}\right) \\
& \times(-\bar{Y}(k)-\xi(k)+V(k))]^{T} P^{-1}(k) \\
& \times\left[\bar{\Theta}_{G}(k-1)+P(k) \Phi\left(k, \widehat{\Theta}_{G}\right)\right. \\
& \times(-\bar{Y}(k)-\xi(k)+V(k))] .
\end{aligned}
$$

So

$$
\begin{aligned}
S(k)= & \bar{\Theta}_{G}^{T}(k-1) P^{-1}(k) \bar{\Theta}_{G}(k-1) \\
& +2 \bar{\Theta}_{G}^{T}(k-1) \Phi\left(k, \widehat{\Theta}_{G}\right) \\
& \times[-\bar{Y}(k)-\xi(k)+V(k)] \\
& +\Phi^{T}\left(k, \widehat{\Theta}_{G}\right) P(k) \Phi\left(k, \widehat{\Theta}_{G}\right) \\
& \times[-\bar{Y}(k)-\xi(k)+V(k)] \\
& \times[-\bar{Y}(k)-\xi(k)+V(k)]^{T} .
\end{aligned}
$$

Substituting $P^{-1}(k)$ by (30), we get

$$
\begin{aligned}
S(k)= & \bar{\Theta}_{G}^{T}(k-1)\left[P^{-1}(k-1)\right. \\
& \left.+\Phi\left(k, \widehat{\Theta}_{G}\right) \Phi^{T}\left(k, \widehat{\Theta}_{G}\right)\right] \\
& \times \bar{\Theta}_{G}(k-1)+2 \bar{\Theta}_{G}^{T}(k-1) \Phi\left(k, \widehat{\Theta}_{G}\right) \\
& \times[-\bar{Y}(k)-\xi(k)+V(k)] \\
& +\Phi^{T}\left(k, \widehat{\Theta}_{G}\right) P(k) \Phi\left(k, \widehat{\Theta}_{G}\right) \\
& \times[-\bar{Y}(k)-\xi(k)+V(k)] \\
& \times[-\bar{Y}(k)-\xi(k)+V(k)]^{T} .
\end{aligned}
$$

Then,

$$
\begin{aligned}
S(k)= & S(k-1)+\bar{Y}(k) \bar{Y}(k)^{T} \\
& -2 \bar{Y}(k) \bar{Y}(k)^{T}-2 \bar{Y}(k) \xi^{T}(k) \\
& +2 \bar{Y}(k) V^{T}(k)+\Phi^{T}\left(k, \widehat{\Theta}_{G}\right) P(k) \Phi \\
& \times\left(k, \widehat{\Theta}_{G}\right) \bar{Y}(k) \bar{Y}(k)^{T} \\
& -2 \Phi^{T}\left(k, \widehat{\Theta}_{G}\right) P(k) \Phi\left(k, \widehat{\Theta}_{G}\right) \bar{Y}(k) \\
& \times(-\xi(k)+V(k))^{T}+\Phi^{T}\left(k, \widehat{\Theta}_{G}\right) \\
& \times P(k) \Phi\left(k, \widehat{\Theta}_{G}\right)\left(\xi(k) \xi(k)^{T}+V(k) V(k)^{T}\right. \\
& \left.\quad-2 \xi(k) V^{T}(k)\right) .
\end{aligned}
$$


Step 1. Data acquisition $\{U(k), Y(k)\}$ and initialization: set $\widehat{\Theta}_{G}=\Theta_{G_{0}}$ and $P=\beta I$ where $\beta$ is a scalar and $I$ is the identity matrix of size $\left(p, p\left(n_{a}+n_{b}+n_{c}+1\right)\right)$ and $k=0$,

Step 2. Increment $k$ and construct the observation vector $\varphi(k, \widetilde{D})$, the generalized observation vector $\Phi^{T}\left(k, \widehat{\Theta}_{G}\right)$ using $(9)$ and $(16)$,

Step 3. Estimate $\widehat{\Theta}_{G}$ using the developed identification method:

$\Upsilon(k)=Y(k)-\widehat{\Theta}^{T}(k-1) \varphi(k, \widetilde{D})$

$P(k)=P(k-1)-\frac{P(k-1) \Phi\left(k, \widehat{\Theta}_{G}\right) \Phi^{T}\left(k, \widehat{\Theta}_{G}\right) P(k-1)}{1+\Phi^{T}\left(k, \widehat{\Theta}_{G}\right) P(k-1) \Phi\left(k, \widehat{\Theta}_{G}\right)}$

$\widehat{\Theta}_{G}(k)=\widehat{\Theta}_{G}(k-1)+P(k) \Phi\left(k, \widehat{\Theta}_{G}\right) \Upsilon(k)$

Step 4. Return to Step 2 until $k=N$ where $N$ is the number of input/output data.

Algorithm 1

It follows from (43) that

$$
\begin{aligned}
S(k)= & S(k-1) \\
& -\left[1-\Phi^{T}\left(k, \widehat{\Theta}_{G}\right) P(k) \Phi\left(k, \widehat{\Theta}_{G}\right)\right] \\
& \times \bar{Y}(k) \bar{Y}(k)^{T}+\Phi^{T}\left(k, \widehat{\Theta}_{G}\right) \\
& \times P(k) \Phi\left(k, \widehat{\Theta}_{G}\right) V(k) V(k)^{T} \\
& +2\left[1-\Phi^{T}\left(k, \widehat{\Theta}_{G}\right) P(k) \Phi\left(k, \widehat{\Theta}_{G}\right)\right] \\
& \times \bar{Y}(k) V^{T}(k)-2 \\
& \times\left[1-\Phi^{T}\left(k, \widehat{\Theta}_{G}\right) P(k) \Phi\left(k, \widehat{\Theta}_{G}\right)\right] \\
& \times \bar{Y}(k) \xi^{T}(k)+\Phi^{T}\left(k, \widehat{\Theta}_{G}\right) P(k) \Phi \\
& \times\left(k, \widehat{\Theta}_{G}\right) \xi(k) \xi(k)^{T} \\
& -2 \Phi^{T}\left(k, \widehat{\Theta}_{G}\right) P(k) \Phi \\
& \times\left(k, \widehat{\Theta}_{G}\right) \xi(k) V^{T}(k) .
\end{aligned}
$$

We have

$$
\begin{aligned}
& -2\left[1-\Phi^{T}\left(k, \widehat{\Theta}_{G}\right) P(k) \Phi\left(k, \widehat{\Theta}_{G}\right)\right] \\
& \times \bar{Y}(k) \xi^{T}(k) \\
& =\left[1-\Phi^{T}\left(k, \widehat{\Theta}_{G}\right) P(k) \Phi\left(k, \widehat{\Theta}_{G}\right)\right] \\
& \quad \times \bar{Y}(k) \bar{Y}(k)^{T} \\
& +\left[1-\Phi^{T}\left(k, \widehat{\Theta}_{G}\right) P(k) \Phi\left(k, \widehat{\Theta}_{G}\right)\right] \\
& \quad \times \xi(k) \xi(k)^{T}
\end{aligned}
$$

$$
\begin{aligned}
& -\left[1-\Phi^{T}\left(k, \widehat{\Theta}_{G}\right) P(k) \Phi\left(k, \widehat{\Theta}_{G}\right)\right] \\
& \times(\bar{Y}(k)+\xi(k))(\bar{Y}(k)+\xi(k))^{T},
\end{aligned}
$$

and the use of the relation

$$
\begin{aligned}
1- & \Phi^{T}\left(k, \widehat{\Theta}_{G}\right) P(k) \Phi\left(k, \widehat{\Theta}_{G}\right) \\
& =\left[1+\Phi^{T}\left(k, \widehat{\Theta}_{G}\right) P(k-1) \Phi\left(k, \widehat{\Theta}_{G}\right)\right]^{-1} \geqslant 0
\end{aligned}
$$

leads to

$$
\begin{aligned}
S(k) \leqslant & S(k-1)+\Phi^{T}\left(k, \widehat{\Theta}_{G}\right) P(k) \Phi \\
& \times\left(k, \widehat{\Theta}_{G}\right) V(k) V(k)^{T}+2 \\
& \times\left[1-\Phi^{T}\left(k, \widehat{\Theta}_{G}\right) P(k) \Phi\left(k, \widehat{\Theta}_{G}\right)\right] \\
& \times \bar{Y}(k) V^{T}(k)+\xi(k) \xi(k)^{T} \\
& -2 \Phi^{T}\left(k, \widehat{\Theta}_{G}\right) P(k) \Phi\left(k, \widehat{\Theta}_{G}\right) \xi(k) V^{T}(k) .
\end{aligned}
$$

Since $\bar{Y}(k), \Phi^{T}\left(k, \widehat{\Theta}_{G}\right) P(k) \Phi\left(k, \widehat{\Theta}_{G}\right), \xi(k)$, and $S(k-1)$ are uncorrelated with $V(k)$, taking the conditional expectation on both sides of (47) and using (A3) and (A4), we obtain

$$
E[S(k)] \leqslant S(k-1)+2 \Phi^{T}\left(k, \widehat{\Theta}_{G}\right) P(k) \Phi\left(k, \widehat{\Theta}_{G}\right) \Sigma+\beta .
$$

Define

$$
W(k):=\frac{S(k)}{\left[\operatorname{Ln}\left|P^{-1}(k)\right|\right]^{c}} .
$$


Since $\left[\operatorname{Ln}\left|P^{-1}(k)\right|\right]^{c}$ is nondecreasing, we have

$$
\begin{aligned}
E[W(k)] \leqslant & \frac{S(k-1)}{\left[\operatorname{Ln}\left|P^{-1}(k)\right|\right]^{c}} \\
& +\frac{2 \Phi^{T}\left(k, \widehat{\Theta}_{G}\right) P(k) \Phi\left(k, \widehat{\Theta}_{G}\right)}{\left[\operatorname{Ln}\left|P^{-1}(k)\right|\right]^{c}} \Sigma \\
& +\frac{\beta}{\left[\operatorname{Ln}\left|P^{-1}(k)\right|\right]^{c}} \\
\leqslant & W(k-1) \\
& +\frac{2 \Phi^{T}\left(k, \widehat{\Theta}_{G}\right) P(k) \Phi\left(k, \widehat{\Theta}_{G}\right)}{\left[\operatorname{Ln}\left|P^{-1}(k)\right|\right]^{c}} \Sigma \\
& +\frac{\beta}{\left[\operatorname{Ln}\left|P^{-1}(k)\right|\right]^{c}} .
\end{aligned}
$$

Using this property $\sum_{i=1}^{\infty}\left(\Phi^{T}\left(i, \widehat{\Theta}_{G}\right) P(i) \Phi\left(i, \widehat{\Theta}_{G}\right) /[\operatorname{Ln} \mid\right.$ $\left.\left.P^{-1}(i) \mid\right]^{c}\right)<\infty$ (the proof in the same way as the proof of Lemma 1 in [30]), we can see that the sum of the right-hand second term of equation (50) for $k$ from 1 to $\infty$ is finite. Applying the martingale convergence theorem [30] to the previous inequality, we conclude that $W(k)$ converges a.s. to a finite random variable, say $W_{0}$; that is,

$$
W(k)=\frac{S(k)}{\left[\operatorname{Ln}\left|P^{-1}(k)\right|\right]^{c}} \longrightarrow W_{0}<\infty
$$

is equivalent to

$$
S(k)=O\left(\left[\operatorname{Ln}\left|P^{-1}(k)\right|\right]^{c}\right) .
$$

From the definition of $V(k)$, we obtain

$$
\left\|\bar{\Theta}_{G}\right\| \leqslant \frac{\bar{\Theta}_{G}(k)^{T} P^{-1}(k) \bar{\Theta}_{G}(k)}{\lambda_{\min }\left[P^{-1}(k)\right]}=\frac{S(k)}{\lambda_{\min }\left[P^{-1}(k)\right]} .
$$

Now, let us define the matrix trace

$$
r(k)=\operatorname{tr}\left[P^{-1}(k)\right] .
$$

It follows that:

$$
\begin{gathered}
\left|P^{-1}(k)\right| \leqslant r^{n}(k), \\
r(k) \leqslant n \lambda_{\max }\left|P^{-1}(k)\right|, \\
\operatorname{Ln}\left|P^{-1}(k)\right|=O(\operatorname{Ln} r(k))=O\left(\operatorname{Ln} \lambda_{\max }\left|P^{-1}(k)\right|\right) .
\end{gathered}
$$

We obtain, finally,

$$
\begin{aligned}
\left\|\widehat{\Theta}_{G}-\Theta_{G}\right\|^{2} & =O\left(\frac{\left[\operatorname{Ln}\left|P^{-1}(k)\right|\right]^{c}}{\lambda_{\min }\left|P^{-1}(k)\right|}\right) \\
& =O\left(\frac{[\operatorname{Ln} r(k)]^{c}}{\lambda_{\min }\left|P^{-1}(k)\right|}\right) \\
& =O\left(\frac{\left[\lambda_{\max }\left|P^{-1}(k)\right|\right]^{c}}{\lambda_{\min }\left|P^{-1}(k)\right|}\right) .
\end{aligned}
$$

4.2. Lemma. For the estimate (22) with the assumption (A4), the following proprieties hold.

(P1) $\widehat{\Theta}_{G}$ is an unbiased estimate of $\Theta_{G}$.

(P2) The covariance matrix of $\widehat{\Theta}_{G}$ is given by

$$
\begin{aligned}
& E\left[\left(\widehat{\Theta}_{G}-\Theta_{G}\right)\left(\widehat{\Theta}_{G}-\Theta_{G}\right)^{T}\right] \\
& =\left(\sum_{i=0}^{k} \Phi\left(i, \Theta_{G}\right) \Phi\left(i, \Theta_{G}\right)^{T}\right)^{-1} \Sigma,
\end{aligned}
$$

where

$$
\Sigma=\left(\begin{array}{cccc}
\sigma_{1}^{2} & 0 & \cdots & 0 \\
0 & \sigma_{2}^{2} & \ddots & \vdots \\
\vdots & \ddots & \ddots & 0 \\
0 & \cdots & 0 & \sigma_{p}^{2}
\end{array}\right)
$$

Proof. If we replace (8) in (22), we have

$$
\begin{aligned}
\widehat{\Theta}_{G}= & {\left[\sum_{i=0}^{k} \Phi\left(i, \Theta_{G}\right) \Phi^{T}\left(i, \Theta_{G}\right)\right]^{-1} } \\
& \times \sum_{i=0}^{k} \Phi\left(i, \Theta_{G}\right)\left[\Theta^{T} \varphi(i, D)+V(i)+\psi\right] .
\end{aligned}
$$

Then,

$$
\begin{aligned}
\widehat{\Theta}_{G}= & {\left[\sum_{i=0}^{k} \Phi\left(i, \Theta_{G}\right) \Phi^{T}\left(i, \Theta_{G}\right)\right]^{-1} } \\
& \times \sum_{i=0}^{k} \Phi\left(i, \Theta_{G}\right)\left[\Theta^{T} \varphi(i, D)+\psi\right] \\
& +\left[\sum_{i=0}^{k} \Phi\left(i, \Theta_{G}\right) \Phi^{T}\left(i, \Theta_{G}\right)\right]^{-1} \\
& \times \sum_{i=0}^{k} \Phi\left(i, \Theta_{G}\right) V(i) .
\end{aligned}
$$

So

$$
\begin{aligned}
E\left[\widehat{\Theta}_{G}\right]=\Theta_{G}+E( & {\left[\sum_{i=0}^{k} \Phi\left(i, \Theta_{G}\right) \Phi^{T}\left(i, \Theta_{G}\right)\right]^{-1} } \\
& \left.\times \sum_{i=0}^{k} \Phi\left(i, \Theta_{G}\right) V(i)\right) .
\end{aligned}
$$

Since $V(i)$ is uncorrelated with the elements of $\Phi\left(i, \Theta_{G}\right)(13)$, then

$$
E\left[\widehat{\Theta}_{G}\right]=\Theta_{G}
$$

which proves $(\mathrm{P} 1)$. 
Consider the first-order Taylor series expansion around the real matrix of $\Theta_{G}$ :

$$
\frac{\partial J\left(k, \widehat{\Theta}_{G}\right)}{\partial \widehat{\Theta}_{G}}=\frac{\partial J\left(k, \Theta_{G}\right)}{\partial \Theta_{G}}+\frac{\partial^{2} J\left(k, \Theta_{G}\right)}{\partial \Theta_{G}^{2}}\left(\widehat{\Theta}_{G}-\Theta_{G}\right) .
$$

Since $\partial J\left(k, \widehat{\Theta}_{G}\right) / \partial \widehat{\Theta}_{G}=0$, it derives from (63) that

$$
\begin{aligned}
\left(\widehat{\Theta}_{G}\right. & \left.-\Theta_{G}\right)\left(\widehat{\Theta}_{G}-\Theta_{G}\right)^{T} \\
= & {\left[\frac{\partial^{2} J\left(k, \Theta_{G}\right)}{\partial \Theta_{G}^{2}}\right]^{-1} \frac{\partial J\left(k, \Theta_{G}\right)}{\partial \Theta_{G}} } \\
& \times\left[\frac{\partial J\left(k, \Theta_{G}\right)}{\partial \Theta_{G}}\right]^{T}\left[\left(\frac{\partial^{2} J\left(k, \Theta_{G}\right)}{\partial \Theta_{G}^{2}}\right)^{-1}\right]^{T} .
\end{aligned}
$$

The second partial derivative of the criterion with respect to the generalized matrix is

$$
\frac{\partial^{2} J\left(k, \Theta_{G}\right)}{\partial \Theta_{G}^{2}}=\sum_{i=0}^{k}\left(\Upsilon(i) \frac{\partial^{2} \Upsilon(i)}{\partial^{2} \Theta_{G}}-\frac{\partial \Upsilon(i)}{\partial \Theta_{G}} \Phi\left(i, \Theta_{G}\right)\right) .
$$

So

$$
\frac{\partial^{2} J\left(k, \Theta_{G}\right)}{\partial \Theta_{G}^{2}}=\sum_{i=0}^{k}\left(\Upsilon(i) \frac{\partial^{2} \Upsilon(i)}{\partial^{2} \Theta_{G}}+\Phi\left(i, \Theta_{G}\right) \Phi^{T}\left(i, \Theta_{G}\right)\right) .
$$

The use of the small residual algorithms [31] leads to neglect the following term, then:

$$
\sum_{i=0}^{k} \Upsilon(i) \frac{\partial^{2} \Upsilon(i)}{\partial^{2} \Theta_{G}} \longrightarrow 0
$$

Hence, an approach of $\partial^{2} J\left(k, \Theta_{G}\right) / \partial \Theta_{G}^{2}$ is obtained:

$$
\frac{\partial^{2} J\left(k, \Theta_{G}\right)}{\partial \Theta_{G}^{2}} \simeq \sum_{i=0}^{k}\left(\Phi\left(i, \Theta_{G}\right) \Phi^{T}\left(i, \Theta_{G}\right)\right) .
$$

Applying the mean value of $\left(\partial J\left(k, \Theta_{G}\right) / \partial \Theta_{G}\right)\left(\partial J\left(k, \Theta_{G}\right) /\right.$ $\partial \Theta_{G}^{T}$ ), we get:

$$
\begin{aligned}
E & {\left[\frac{\partial J\left(k, \Theta_{G}\right)}{\partial \Theta_{G}} \frac{\partial J\left(k, \Theta_{G}\right)^{T}}{\partial \Theta_{G}}\right] } \\
& =\sum_{i=0}^{k} \Phi\left(i, \Theta_{G}\right) \Phi\left(i, \Theta_{G}\right)^{T} E\left(\Upsilon(i) \Upsilon(i)^{T}\right) .
\end{aligned}
$$

So

$$
E\left[\frac{\partial J\left(k, \Theta_{G}\right)}{\partial \Theta_{G}} \frac{\partial J\left(k, \Theta_{G}\right)^{T}}{\partial \Theta_{G}}\right]=\sum_{i=0}^{k} \Phi\left(i, \Theta_{G}\right) \Phi\left(i, \Theta_{G}\right)^{T} \Sigma .
$$

Then, we have

$$
\begin{aligned}
& E\left[\left(\widehat{\Theta}_{G}-\Theta_{G}\right)\left(\widehat{\Theta}_{G}-\Theta_{G}\right)^{T}\right] \\
& \quad=E\left[\left(\frac{\partial^{2} J}{\partial \Theta_{G}^{2}}\right)^{-1} \sum_{i=0}^{k} \Phi\left(i, \Theta_{G}\right) \Phi\left(i, \Theta_{G}\right)^{T} \Sigma\left[\left(\frac{\partial^{2} J}{\partial \Theta_{G}^{2}}\right)^{-1}\right]^{T}\right] .
\end{aligned}
$$

Finally, we obtain

$$
\begin{aligned}
E & {\left[\left(\widehat{\Theta}_{G}-\Theta_{G}\right)\left(\widehat{\Theta}_{G}-\Theta_{G}\right)^{T}\right] } \\
& =\left(\sum_{i=0}^{k} \Phi\left(i, \Theta_{G}\right) \Phi\left(i, \Theta_{G}\right)^{T}\right)^{-1} \Sigma
\end{aligned}
$$

which proves (P2).

\section{Results}

We now present a simulation example and an experimental validation to illustrate the performance of the proposed approach for the simultaneous identification of time delays and parameter matrices of square multivariable systems.

5.1. Simulation Example. The objective of the simulation is to compare the efficiency of the proposed method (DRLS) with that of the classic recursive least square approach $(R L S)$ [29] which assumes that the delays are a priori known. In fact, we consider the following cases.

Case 1. The output is noise-free and the RLS method uses the true time delays.

Case 2. The output is noise free and the RLS method uses the misestimated time delays.

Case 3. The output is contaminated by additive noise and the RLS method uses the true time delays.

We consider a square linear multivariable discrete time delay system with two inputs and two outputs described by the following equation [32]:

$$
A\left(q^{-1}\right) Y(k)=B\left(q^{-1}\right) \underline{U}+V(k),
$$

where the delayed inputs and the outputs are defined, respectively, by $\underline{U}(k)=\left(\begin{array}{l}u_{1}\left(k-d_{1}\right) \\ u_{2}\left(k-d_{2}\right)\end{array}\right)$ and $Y(k)=\left(\begin{array}{l}y_{1}(k) \\ y_{2}(k)\end{array}\right)$.

The two polynomials matrices $A\left(q^{-1}\right)$ and $B\left(q^{-1}\right)$ are given by

$$
\begin{gathered}
A\left(q^{-1}\right)=\left(\begin{array}{ll}
1 & 0 \\
0 & 1
\end{array}\right)+\left(\begin{array}{cc}
0.9048 & 0 \\
0 & 0.9048
\end{array}\right) q^{-1}, \\
B\left(q^{-1}\right)=\left(\begin{array}{cc}
0.09516 & 0.03807 \\
-0.0297 & 0.0475
\end{array}\right) q^{-1} .
\end{gathered}
$$

The time delay matrix $D$ is given by

$$
D=\left(\begin{array}{ll}
3 & 0 \\
0 & 1
\end{array}\right)
$$




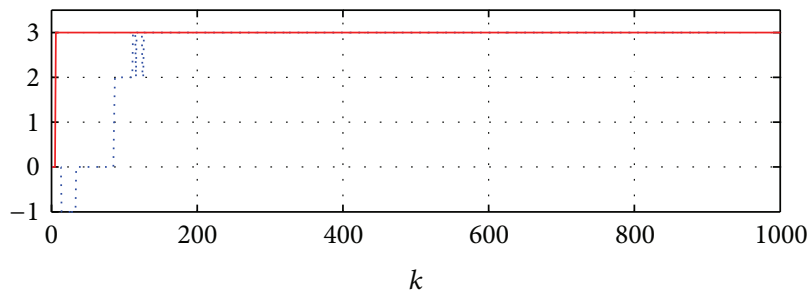

$k$

..... DRLS

- True

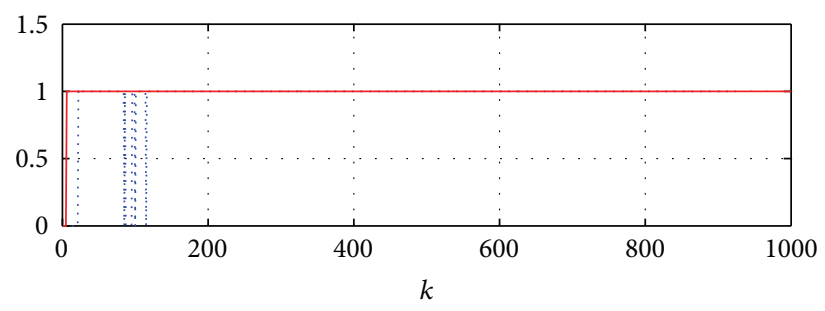

DRLS

(a)

(b)

FIgURE 1: Evolution of the true (-) and the estimated (- -) delays: Case 1.

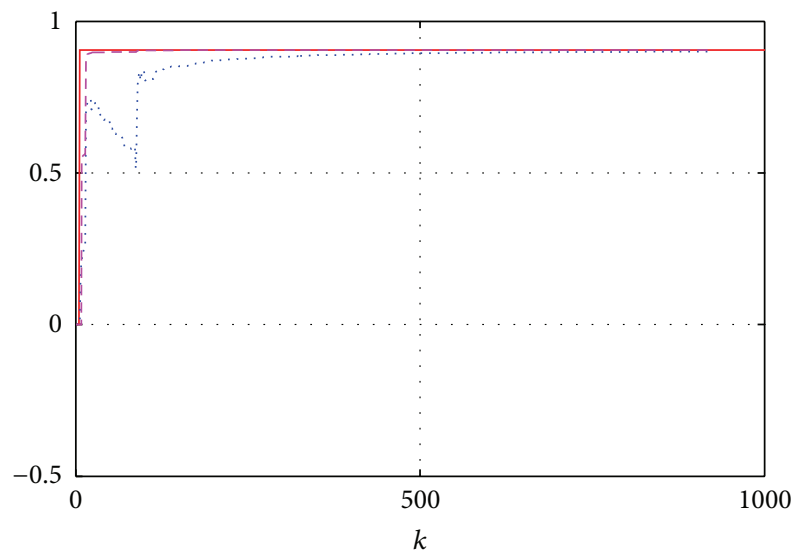

(a)

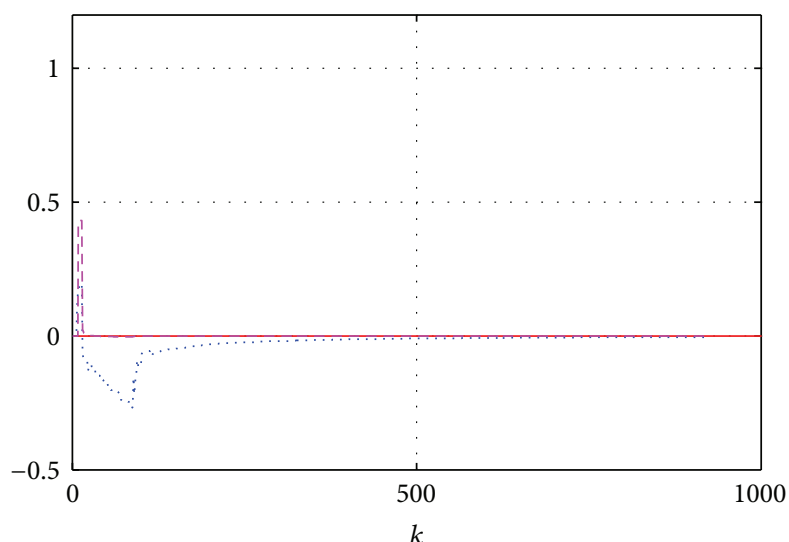

- True

DRLS

-. - RLS

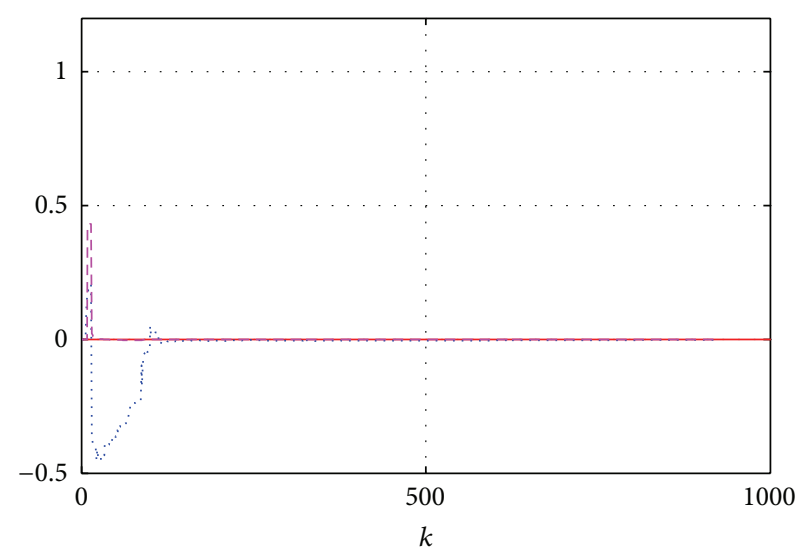

(b)

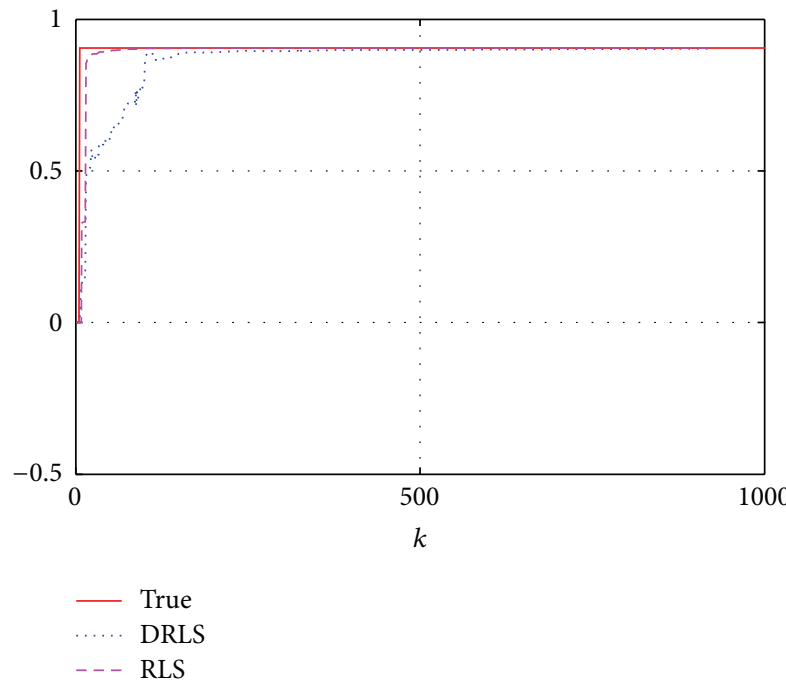

(d)

(c)

Figure 2: Evolution of the true and the estimated parameters: $A_{i}$ : Case 1.

5.1.1. Case 1. The proposed approach (DLSR) and the RLS algorithm are applied to estimate time delays and parameter matrices. The estimation starts with zero initial conditions. The obtained results are illustrated in Table 1 and Figures 1, 2, and 3.
Figures 1-3 show the evolution of the estimated and the true parameter matrices.

A validation of the obtained model is presented in Figures 4 and 5 which show that the estimated outputs track fast and accurately the true outputs. 


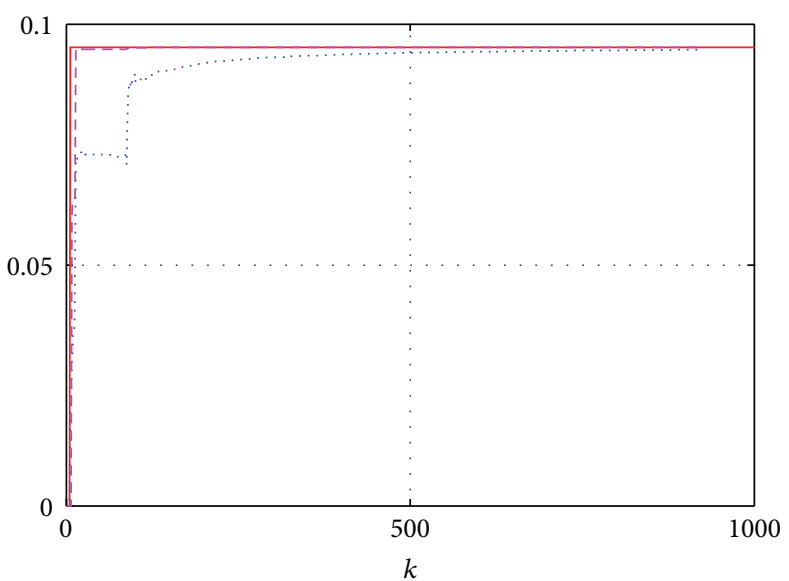

(a)

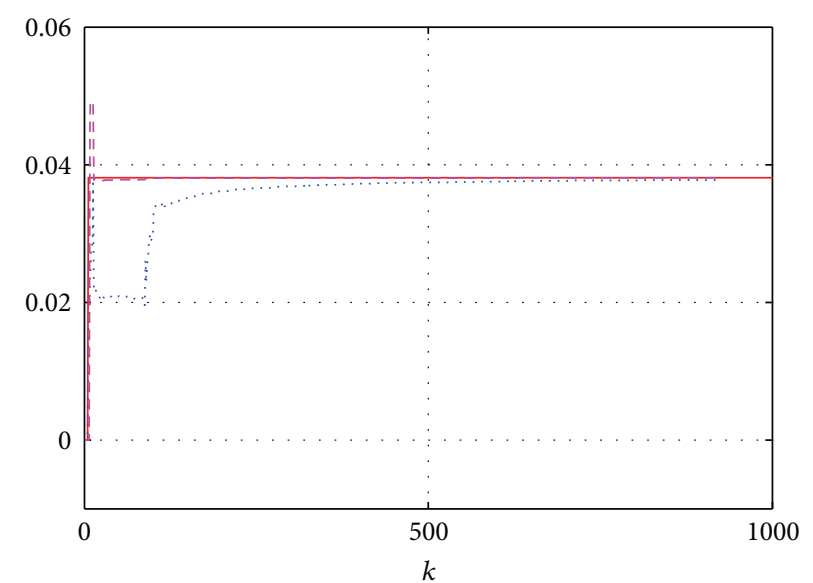

- True

…. DRLS

- - RLS

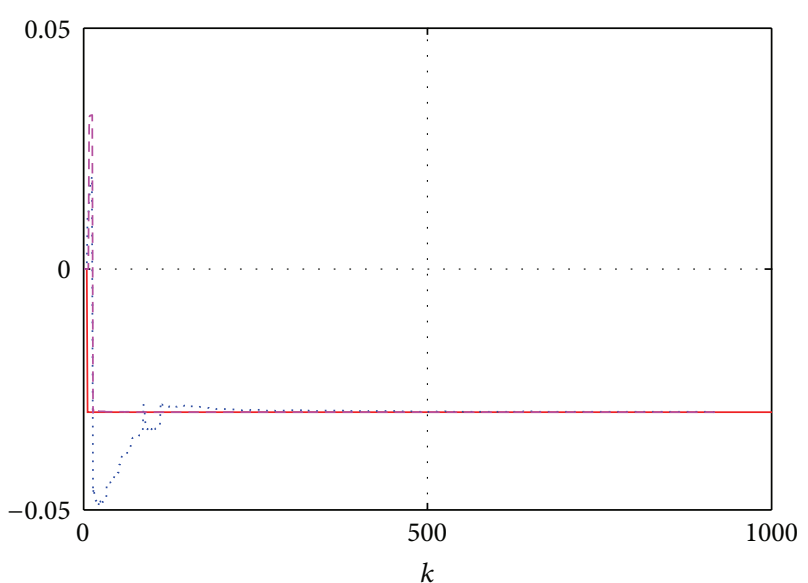

(b)

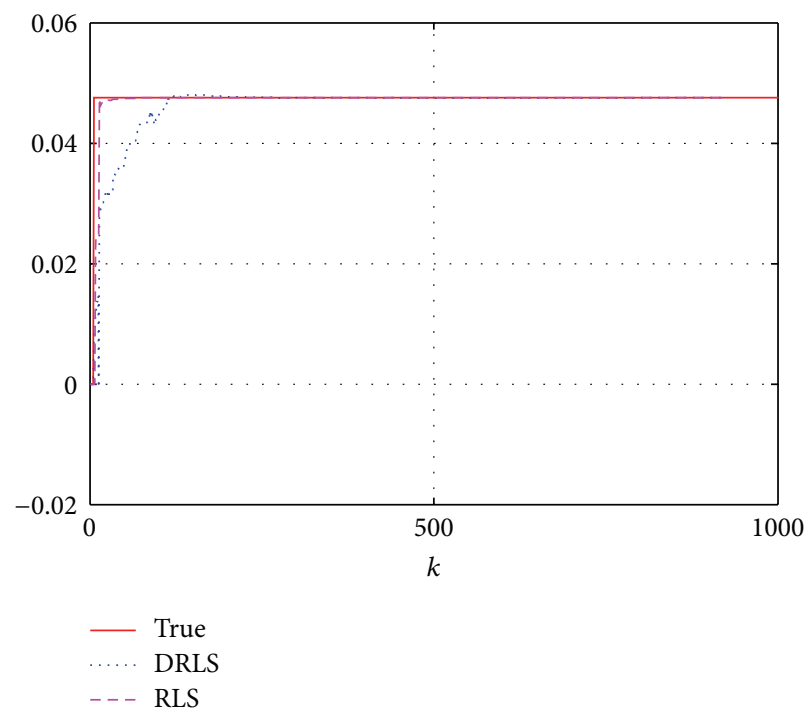

(d)

(c)

Figure 3: Evolution of the true and the estimated parameters: $B_{i}$ : Case 1.

TABLE 1: Simulation results: Case 1.

\begin{tabular}{cccccccc}
\hline & \multicolumn{2}{c}{ True } & \multicolumn{2}{c}{ Developed RLS } & \multicolumn{2}{c}{ RLS } \\
\hline \multirow{2}{*}{$A_{1}$} & 0.9048 & 0 & \multicolumn{2}{c}{0.9} & -0.0022 & 0.9048 & -0.0000 \\
& 0 & 0.9048 & -0.0043 & 0.9021 & -0.0000 & 0.9048 \\
$B_{1}$ & 0.09516 & 0.03807 & 0.0947 & 0.0378 & 0.0952 & 0.0381 \\
& -0.02974 & 0.04758 & -0.0297 & 0.0475 & -0.0297 & 0.0476 \\
& 3 & 0 & 3 & 0 & Known \\
& 0 & 1 & 0 & 1 & \\
\hline
\end{tabular}

5.1.2. Case 2. We apply the proposed approach (DLSR) and the RLS algorithm to estimate time delays and parameter matrices. The RLS algorithm uses misestimated time delays $\left(\begin{array}{ll}2 & 0 \\ 0 & 1\end{array}\right)$. The obtained results are illustrated in Table 2 and Figures 6 and 7 .

Figures 6 and 7 show the evolution of the estimated and the true parameter matrices.
TABLE 2: Simulation results: Case 2.

\begin{tabular}{lccccccc}
\hline & \multicolumn{2}{c}{ True } & \multicolumn{2}{c}{ Developed RLS } & \multicolumn{2}{c}{ RLS } \\
\hline \multirow{2}{*}{$A_{1}$} & 0.9048 & 0 & \multicolumn{2}{c}{0.9} & -0.0022 & 0.5829 & -0.2376 \\
& 0 & 0.9048 & -0.0043 & 0.9021 & -0.1288 & -0.1288 \\
& $B_{1}$ & 0.09516 & 0.03807 & 0.0947 & 0.0378 & 0.0692 & 0.0277 \\
& -0.02974 & 0.04758 & -0.0297 & 0.0475 & 0.0277 & 0.0463 \\
& 3 & 0 & 3 & 0 & 2 & 0 \\
& 0 & 1 & 0 & 1 & 0 & 1 \\
\hline
\end{tabular}

5.1.3. Case 3. The system's output is corrupted by additive zero mean white noises $V(k)=\left[v_{1}(k), v_{2}(k)\right]$ with variances $\sigma_{1}=0.0737, \sigma_{2}=0.086$.

The result of the simulation is given in Table 3 .

Figures 8, 9, and 10 show the evolution of the estimated and the true parameter matrices. 


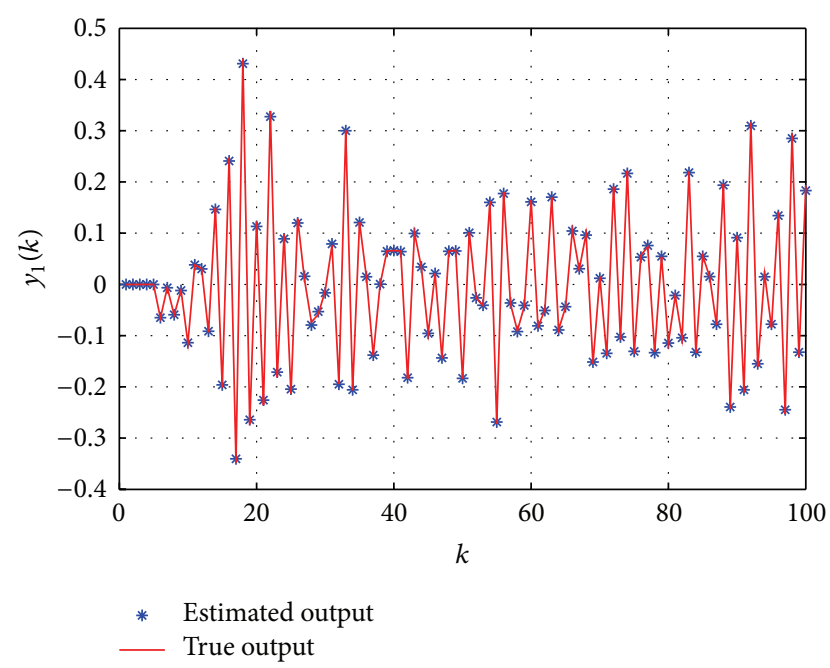

FIGURE 4: The evolution of the true and the estimated outputs $y_{1}(k)$ : Case 1.

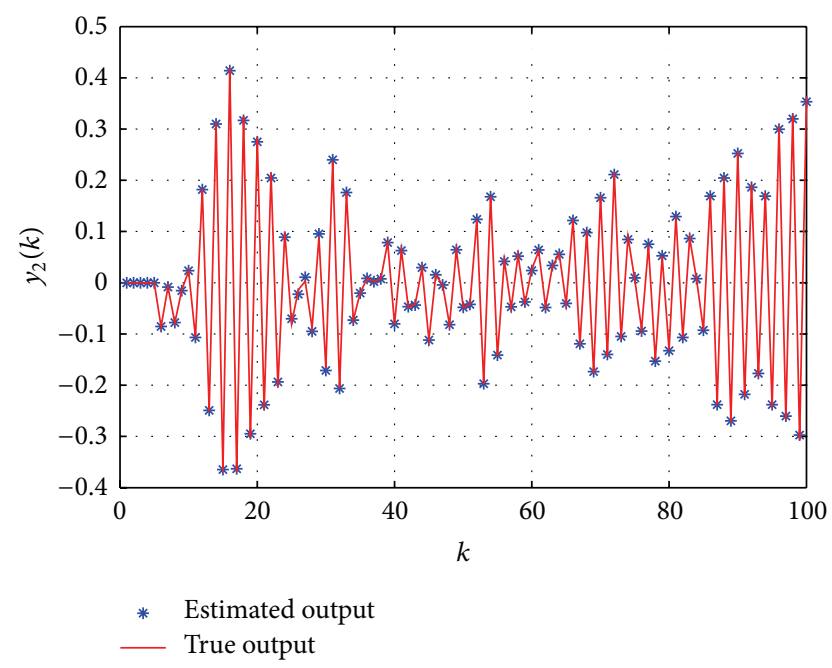

Figure 5: The evolution of the true and the estimated outputs $y_{2}(k)$ : Case 1.

TABLE 3: Simulation results: Case 3.

\begin{tabular}{cccccccc}
\hline & \multicolumn{2}{c}{ True } & \multicolumn{2}{c}{ Developed RLS } & \multicolumn{2}{c}{ RLS } \\
\hline \multirow{2}{*}{$A_{1}$} & 0.9048 & 0 & 0.8949 & -0.0084 & 0.9032 & -0.0016 \\
& 0 & 0.9048 & -0.0084 & 0.9 & 0.0003 & 0.951 \\
$B_{1}$ & 0.09516 & 0.03807 & 0.0941 & 0.073 & 0.0952 & 0.0381 \\
& -0.02974 & 0.04758 & -0.0303 & 0.0476 & -0.0297 & 0.0476 \\
0 & 3 & 0 & 3 & 0 & Known \\
& 0 & 1 & 0 & 1 & \\
\hline
\end{tabular}

A validation of the obtained model is presented in Figures 11 and 12 which show that the estimated outputs track fast and accurately the true outputs.
5.1.4. Observations. Based on Tables 1-3 and Figures 1-12, we observe that

(i) the RLS method gives the better performance when the true time delays are used. However, it poorly performs for misestimated delays;

(ii) the proposed approach converges to the true delays with acceptable speed for the considered cases.

5.2. Experiment Example. The experimental data from a mechanical construction of a CD player arm is considered. The system has two inputs that are forces of the mechanical actuators $(U)$ and two outputs that are related to the tracking accuracy of the $\operatorname{arm}(Y)$.

The data set contains 2048 sample points out of which 1000 were used for the identification procedure and the rest to validate the identified models. The input/output identification signals [33] are given in Figures 13 and 14.

The system is described by the following equation:

$$
A\left(q^{-1}\right) Y(k)=B\left(q^{-1}\right) \underline{U}(k)+V(k),
$$

where $Y(k)$ and $\underline{U}(k)$ are the output and the delayed input of the system at time $k, p=2$ and the orders of $A\left(q^{-1}\right), B\left(q^{-1}\right)$ are $n_{a}=n_{b}=2$.

The estimation starts with zero initial values for the parameter and the time delay matrices. Applying the proposed algorithm, we obtain

$$
\begin{aligned}
\widehat{A}\left(q^{-1}\right)= & \left(\begin{array}{ll}
1 & 0 \\
0 & 1
\end{array}\right)+\left(\begin{array}{cc}
-0.9464 & -0.6600 \\
-0.1263 & -0.5278
\end{array}\right) q^{-1} \\
& +\left(\begin{array}{cc}
0.0809 & 0.5506 \\
-0.1066 & 0.2243
\end{array}\right) q^{-2}, \\
\widehat{B}\left(q^{-1}\right)= & \left(\begin{array}{cc}
-1.4839 & 1.0274 \\
0.2900 & -1.1819
\end{array}\right) q^{-1} \\
& +\left(\begin{array}{cc}
1.2452 & -0.7638 \\
-0.1866 & 0.8853
\end{array}\right) q^{-2} .
\end{aligned}
$$

The estimated time delay matrix is

$$
\widehat{D}=\left(\begin{array}{ll}
0 & 0 \\
0 & 2
\end{array}\right) \text {. }
$$

Another data set is used for the validation test which is illustrated by Figures 15 and 16 .

We can see clearly that the estimated output tracks fast and accurately the true output.

\section{Conclusions}

In this paper, we have addressed the problem of identification of linear discrete time delay multivariable systems. In fact, we have proposed a novel approach for the simultaneous identification of the unknown time delays and the parameter matrices of these systems. The proposed approach consists in constructing a linear-parameter formulation that is used to 


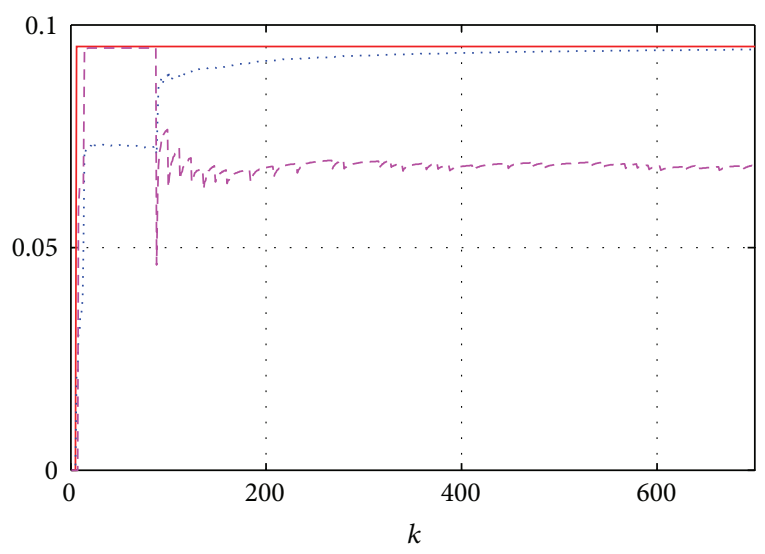

(a)

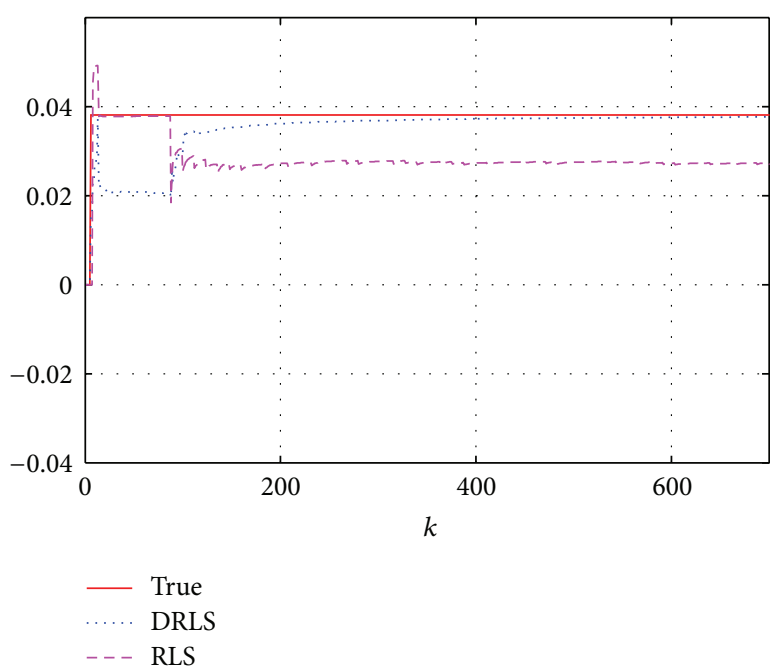

(c)

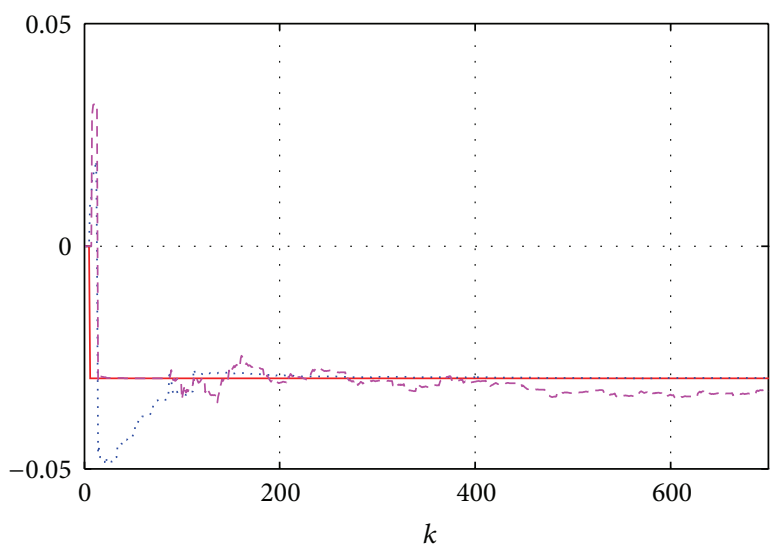

(b)

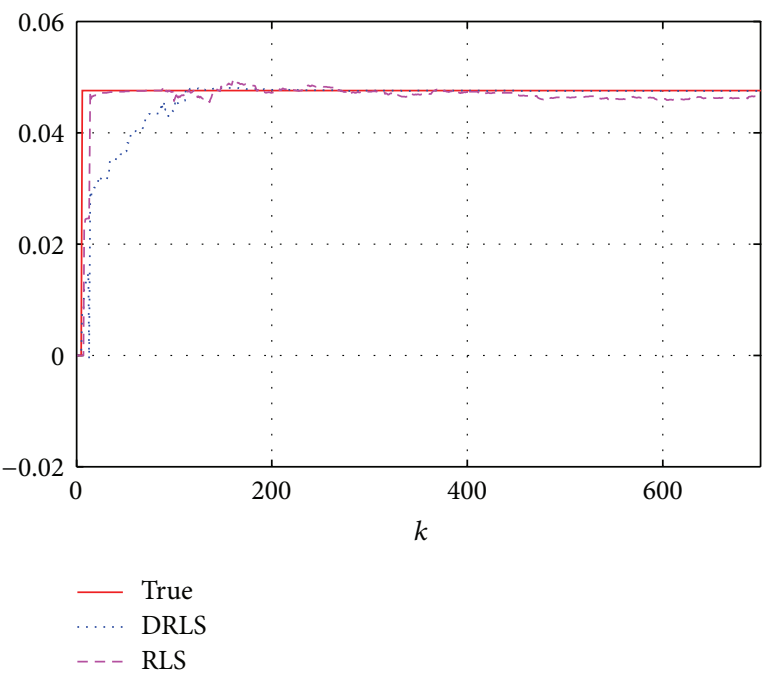

(d)

Figure 6: Evolution of the true and the estimated parameters: $A_{i}$ : Case 2.

estimate the time delays and the polynomial matrices using recursive least square algorithm. The obtained estimates were shown to be unbiased, and an expression for their covariance matrix was given. Numerical simulation and experimental test are presented to demonstrate the performance of the proposed approach.

\section{Appendix}

\section{A. Approximation of $\operatorname{Ln}(q)$}

Let us consider the shift operator and the backward difference given, respectively, by

$$
\begin{gathered}
q u(k)=u(k+1), \\
\Delta u(k)=u(k)-u(k-1) .
\end{gathered}
$$

So

$$
\Delta u(k)=\left(1-q^{-1}\right) u(k) .
$$

We can infer the identity between the shift operator and the backward difference [34], and then

$$
\Delta=1-q^{-1} \text {. }
$$

It is equivalent to

$$
q^{-1}=1-\Delta
$$

Applying the logarithm function of both sides of (A.1), we get

$$
\operatorname{Ln}(q)=-\operatorname{Ln}(1-\Delta)
$$

Using the series expansion of $\operatorname{Ln}(1-x)$, we have

$$
\operatorname{Ln}(q)=\Delta+\frac{1}{2} \Delta^{2}+\frac{1}{3} \Delta^{3}+\cdots .
$$




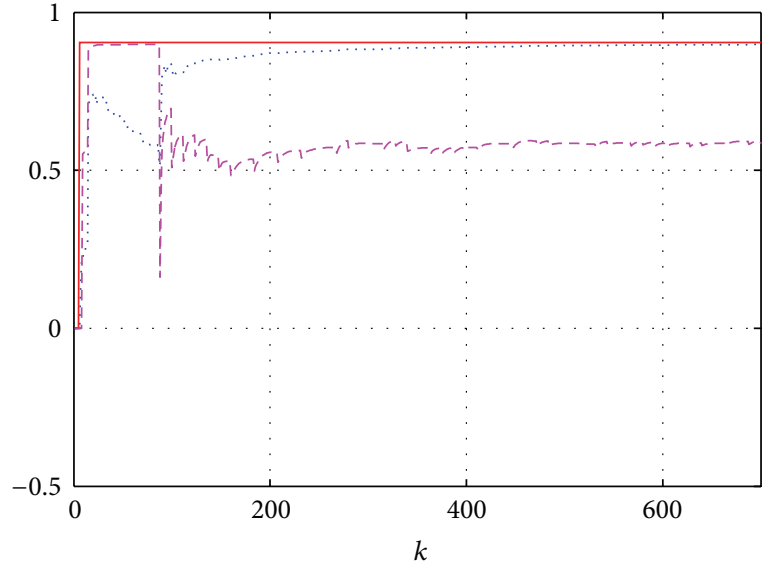

(a)
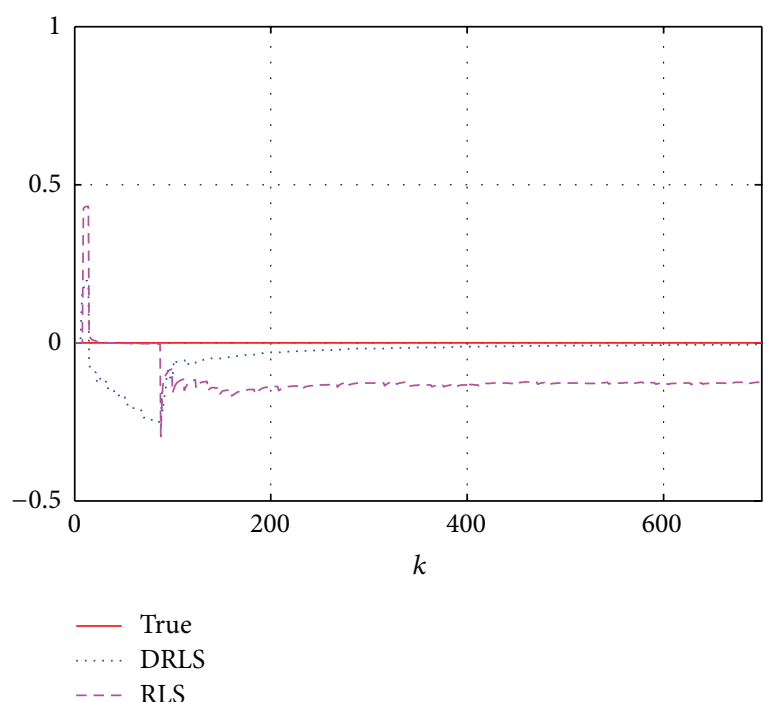

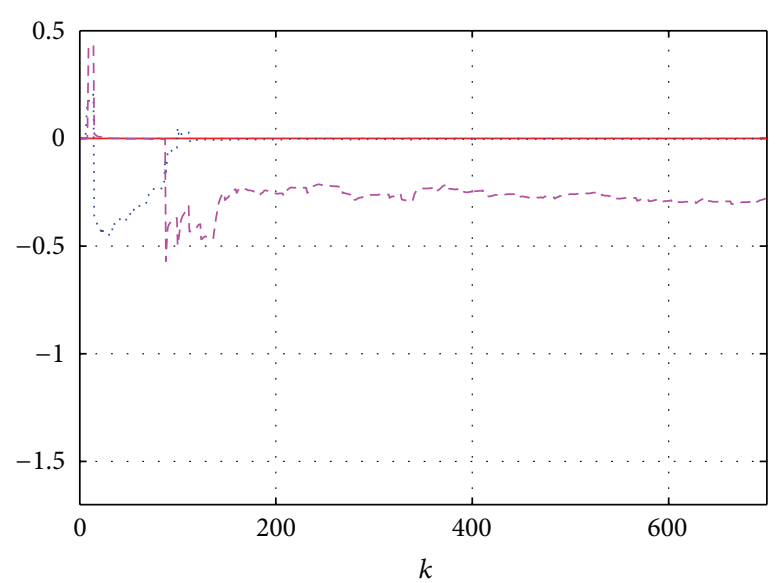

(b)

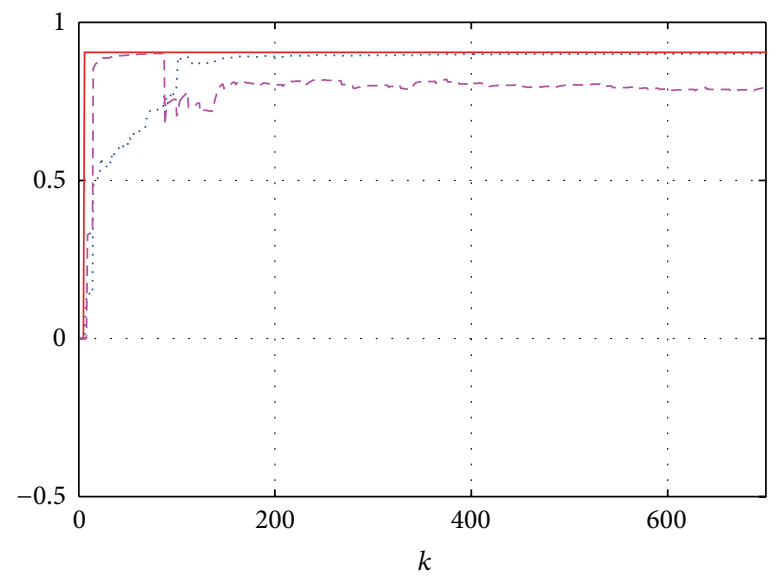

- True DRLS

- - RLS

(c)

(d)

FIgURE 7: Evolution of the true and the estimated parameters: $B_{i}$ : Case 2.

Finally, we use a first-order approximation of the shift operator given by

$$
\operatorname{Ln}(q)=\Delta=1-q^{-1}
$$

\section{B. Expression of $\partial \widehat{\Omega} / \partial \widehat{D}$}

The partial derivative of $\widehat{\Omega}$ with respect to the estimated time delay matrix is given by

$$
\frac{\partial \widehat{\Omega}}{\partial \widehat{D}}=\frac{\partial}{\partial \widehat{D}}\left(\begin{array}{cccc}
q^{-\widehat{d}_{1}} & 0 & \cdots & 0 \\
0 & q^{-\widehat{d}_{2}} & \ddots & \vdots \\
\vdots & \ddots & \ddots & 0 \\
0 & \cdots & 0 & q^{-\widehat{d}_{p}}
\end{array}\right)
$$

It is equivalent to

$$
\frac{\partial \widehat{\Omega}}{\partial \widehat{D}}=\frac{\partial}{\partial \widehat{D}}\left(\begin{array}{cccc}
e^{-\widehat{d}_{1} \operatorname{Ln}(q)} & 0 & \cdots & 0 \\
0 & e^{-\widehat{d}_{2} \operatorname{Ln}(q)} & \ddots & \vdots \\
\vdots & \ddots & \ddots & 0 \\
0 & \cdots & 0 & e^{-\widehat{d}_{p} \operatorname{Ln}(q)}
\end{array}\right) .
$$

Equation (B.2) can be rewritten as

$$
\frac{\partial \widehat{\Omega}}{\partial \widehat{D}}=\frac{\partial}{\partial \widehat{D}} e^{-\widehat{D} \operatorname{Ln}(q)}
$$

We then obtain

$$
\frac{\partial \widehat{\Omega}}{\partial \widehat{D}}=-\operatorname{Ln}(q) \frac{\partial e^{-\widehat{D} \operatorname{Ln}(q)}}{\partial \widehat{D}}=-\operatorname{Ln}(q) \widehat{\Omega}
$$




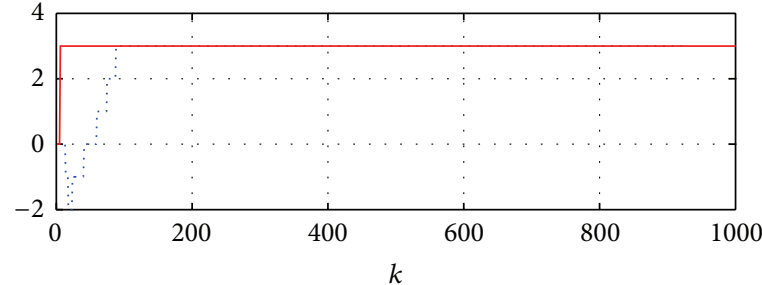

DRLS

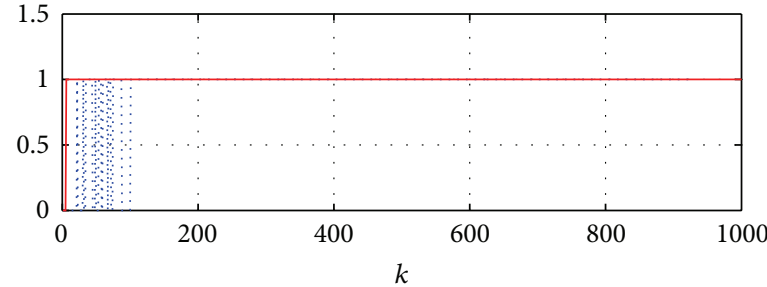

DRLS

(b)

FIGURE 8: Evolution of the true (-) and the estimated (- -) delays: Case 3.

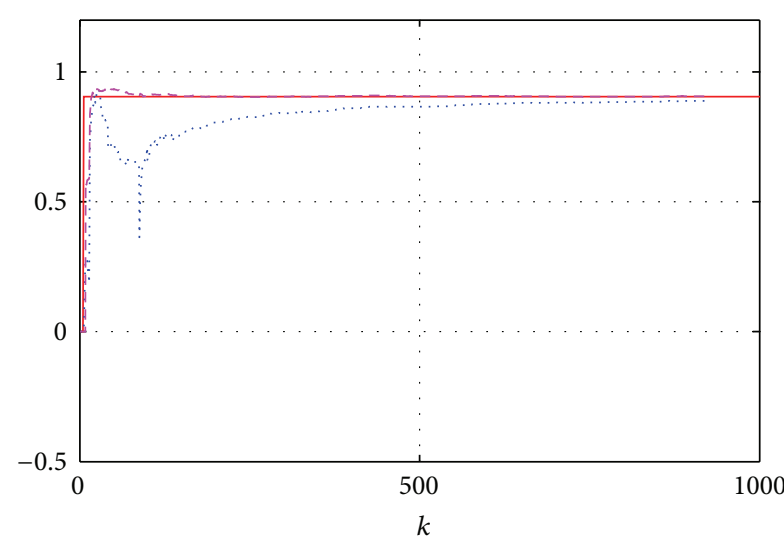

(a)

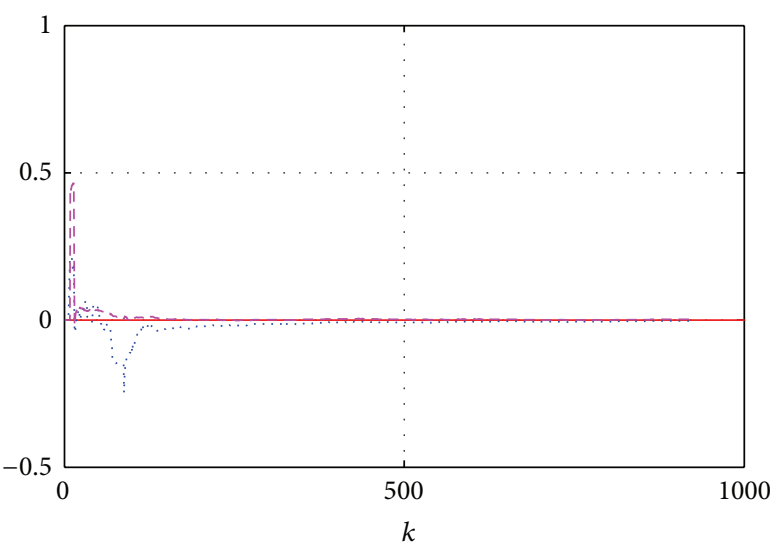

- True

-.... DRLS

- - RLS

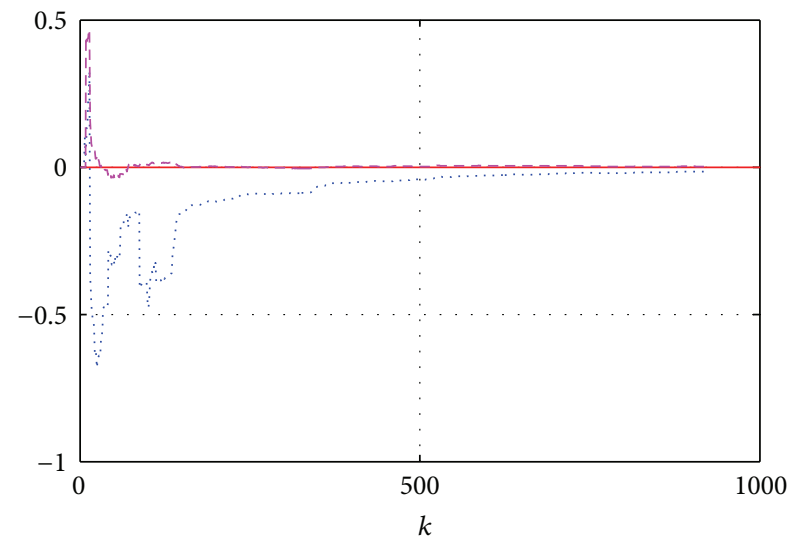

(b)

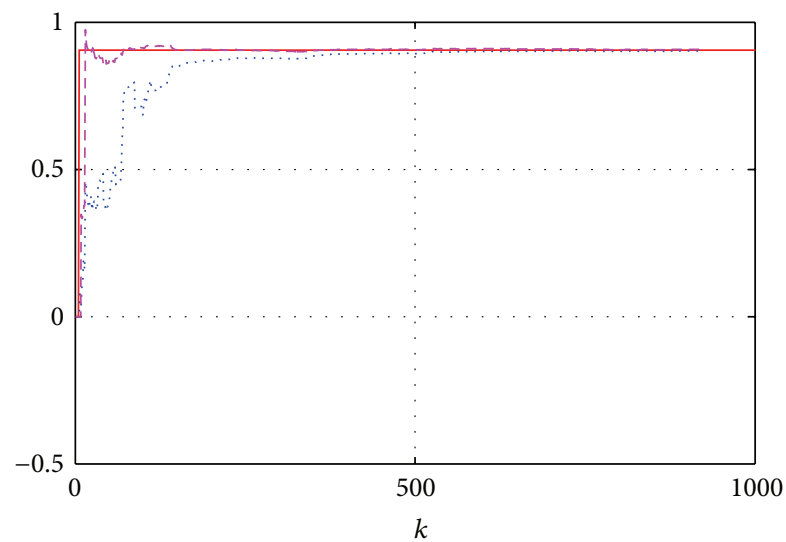

- True

…. DRLS

(c)

(d)

Figure 9: Evolution of the true and the estimated parameters: $A_{i}$ : Case 3.

Using the approximation $\operatorname{Ln}(q) \simeq\left(1-q^{-1}\right)$, we get the partial derivative of $\widehat{\Omega}$ with respect to the estimated time delay matrix:

$$
\frac{\partial \widehat{\Omega}}{\partial \widehat{D}} \simeq-\left(1-q^{-1}\right) \widehat{\Omega}
$$

\section{Expression of $\bar{\rho}(k)$}

We have

$$
\bar{\rho}(k)=\widehat{\Theta}^{T}(k-1) \varphi(k, \widehat{D})-\Theta^{T} \varphi(k, D) .
$$

In the same way as before (see the equations (22) and (21)), the estimated generalized vector parameters $\widehat{\Theta}_{G}$ and the 


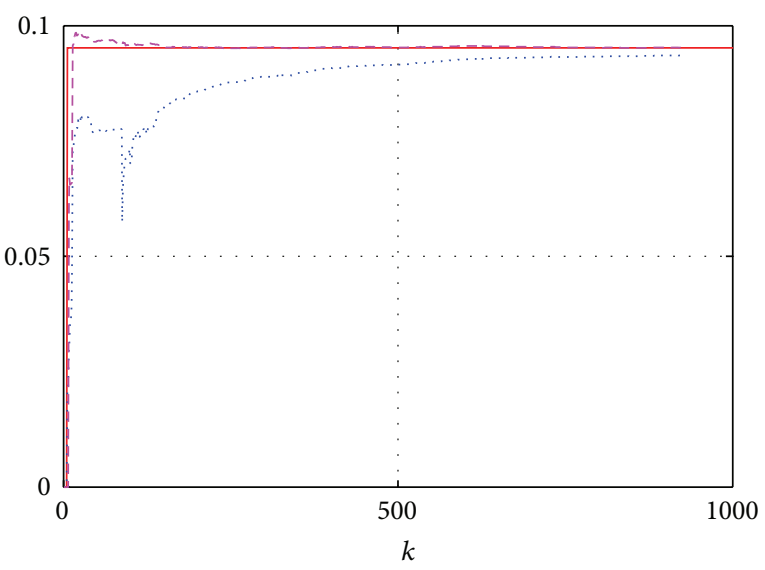

(a)

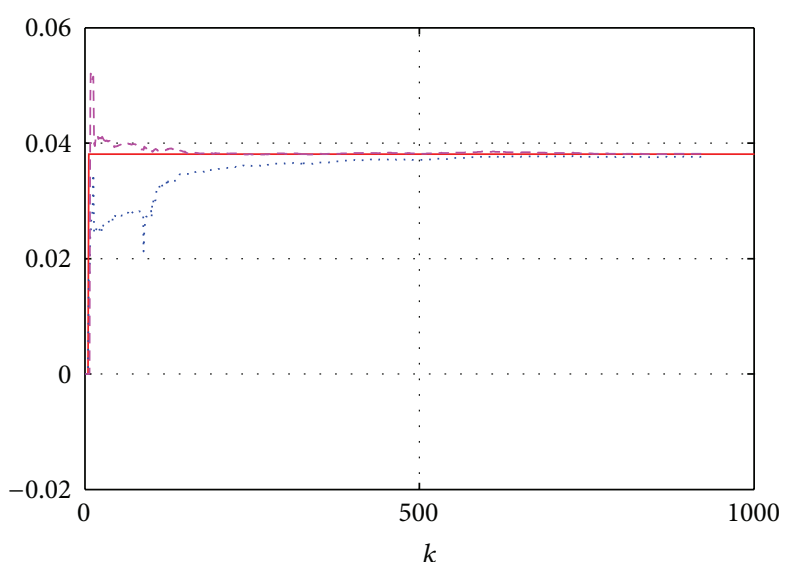

— True

..... DRLS

-.- RLS

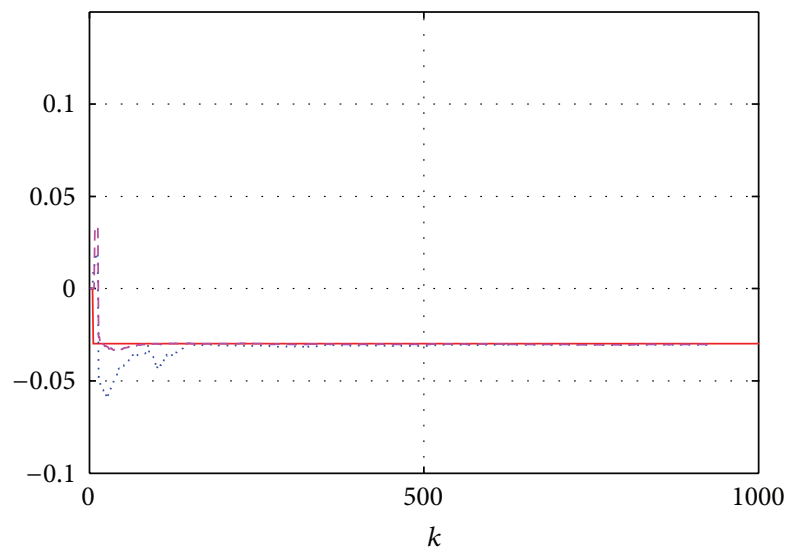

(b)

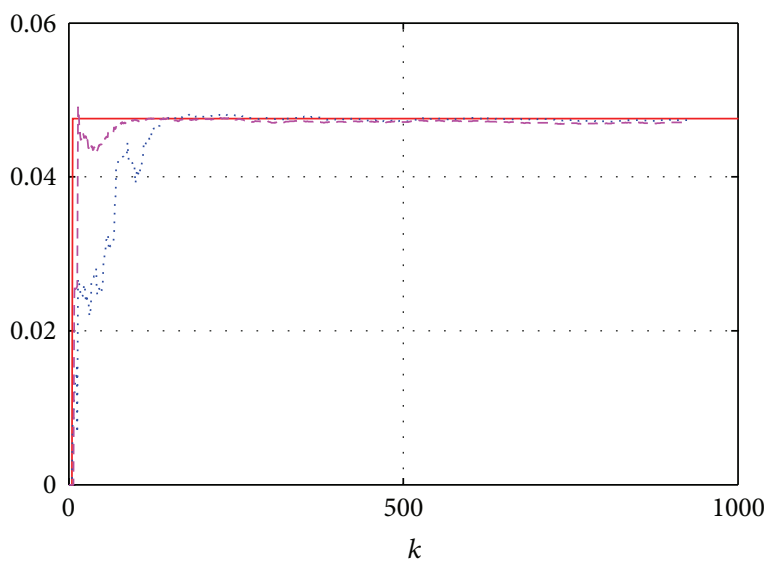

True

…. DRLS

-.- RLS

(c)

(d)

Figure 10: Evolution of the true and the estimated parameters: $B_{i}$ : Case 3.

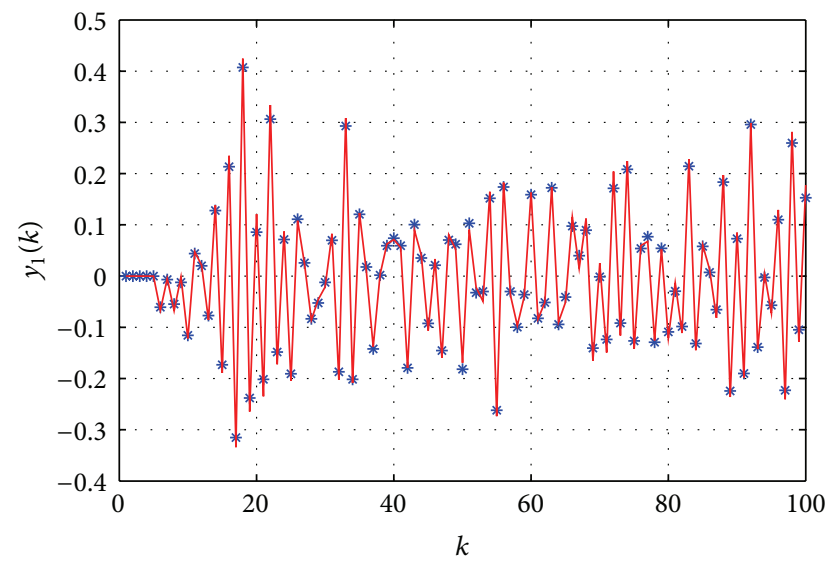

* Estimated output True output

FIGURE 11: The evolution of the true and the estimated outputs $y_{1}(k)$ : Case 3.

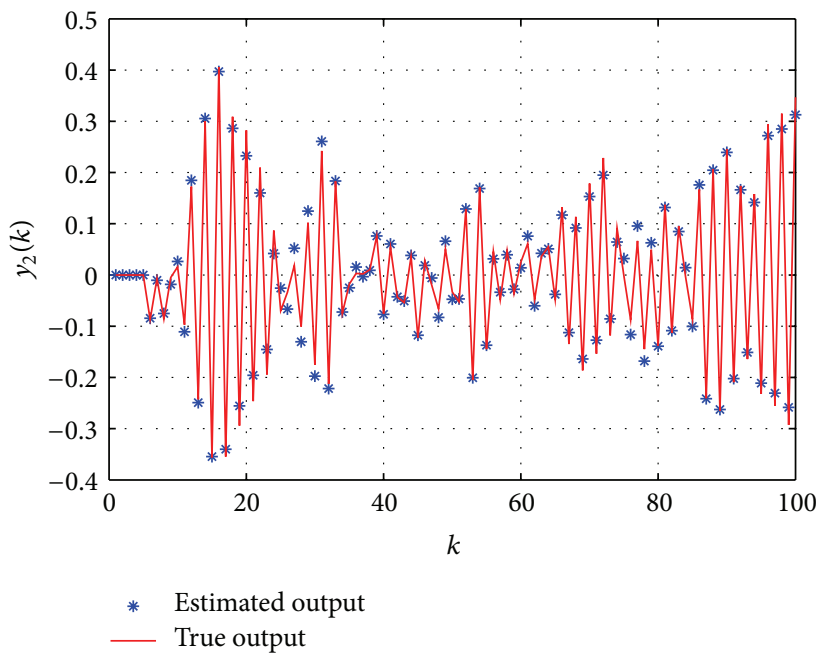

FIGURE 12: The evolution of the true and the estimated outputs $y_{2}(k)$ : Case 3. 


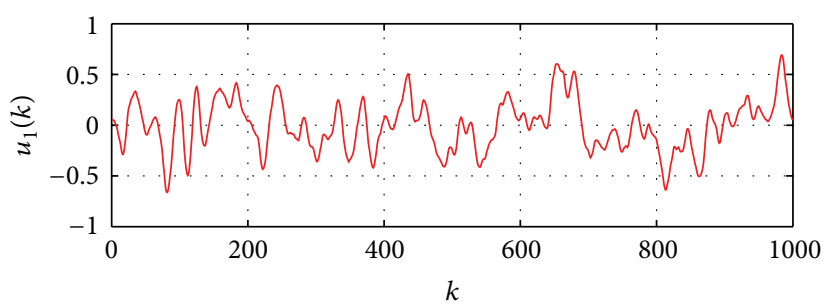

(a)

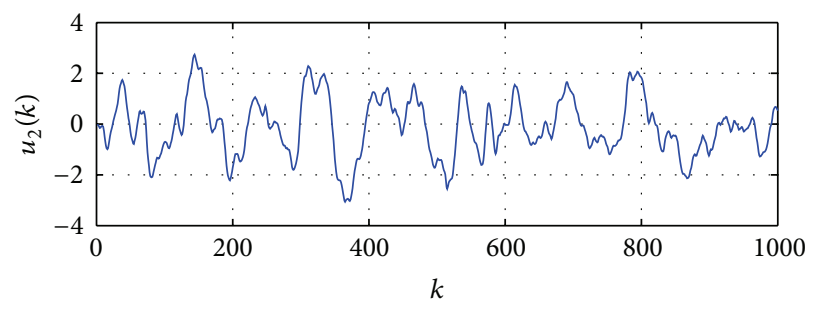

(b)

FIgURE 13: Evolution of input identification signals of CD arm.

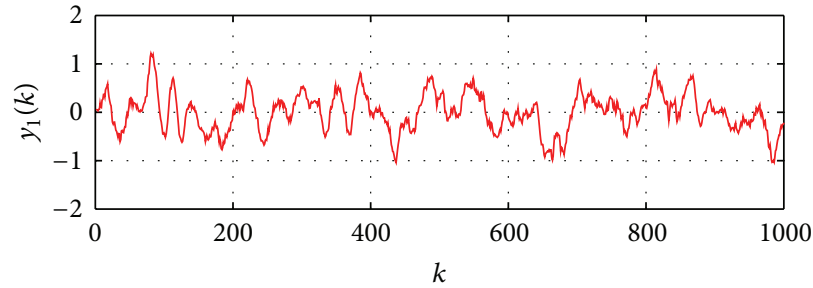

(a)

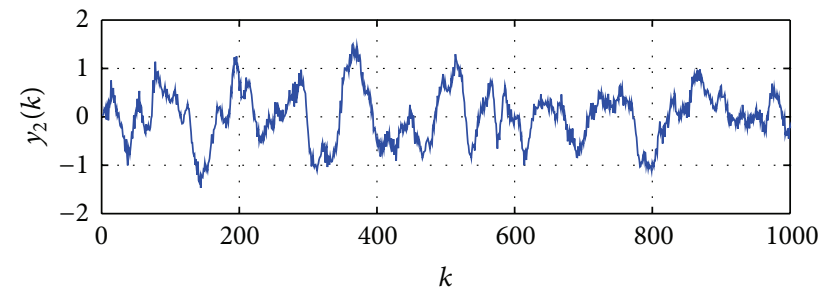

(b)

FIGURE 14: Evolution of output identification signals of CD arm.

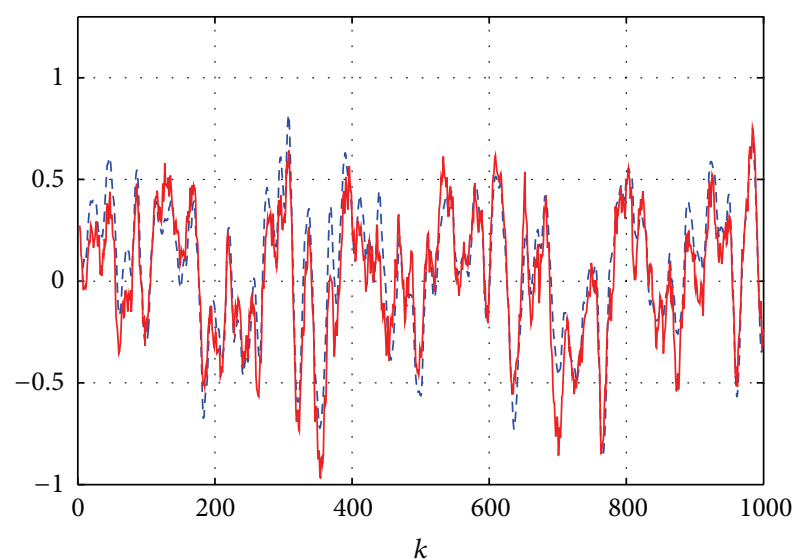

$-\hat{y}_{1}(k)$

$-y_{1}(k)$

FIGURE 15: The evolution of the true (-) and the estimated (- -) outputs.

generalized vector parameters $\Theta_{G}$ can appear after adding and subtracting the appropriate term of the equation (C.1):

$$
\begin{aligned}
\bar{\rho}(k)= & \widehat{\Theta}_{G}^{T}(k-1) \Phi\left(k, \widehat{\Theta}_{G}\right) \\
& +\sum_{i=1}^{n b} \widetilde{D} \widehat{B}_{i} \widehat{\Omega} \Delta U^{T}(k-i) \\
& -\Theta_{G}^{T}(k-1) \Phi\left(k, \Theta_{G}\right) \\
& -\sum_{i=1}^{n b} D B_{i} \Omega \Delta U^{T}(k-i) .
\end{aligned}
$$

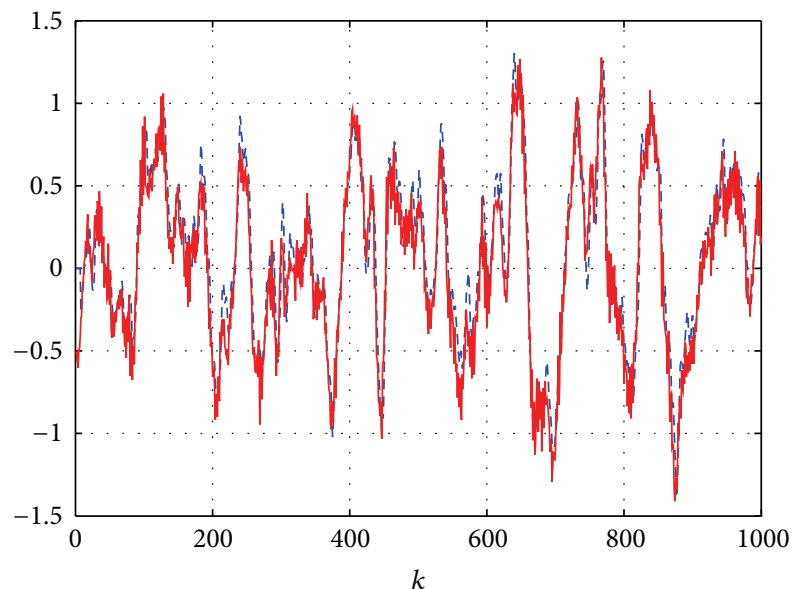

$---\hat{y}_{2}(k)$

$-y_{2}(k)$

FIgURE 16: The evolution of the true (-) and the estimated (- -) outputs.

Adding and subtracting the term $\Theta_{G}(k-1) \Phi\left(k, \widehat{\Theta}_{G}\right)$ from (C.2), we obtain

$$
\begin{aligned}
\bar{\rho}(k)= & \widehat{\Theta}_{G}^{T}(k-1) \Phi\left(k, \widehat{\Theta}_{G}\right) \\
& -\Theta_{G}^{T}(k-1) \Phi\left(k, \Theta_{G}\right)+\alpha \\
& +\Theta_{G}^{T}(k-1) \Phi\left(k, \widehat{\Theta}_{G}\right) \\
& -\Theta_{G}^{T}(k-1) \Phi\left(k, \widehat{\Theta}_{G}\right),
\end{aligned}
$$


where

$$
\begin{aligned}
& \alpha=\sum_{i=1}^{n b} \widehat{D} \widehat{B_{i}} \widehat{\Omega} \Delta U^{T}(k-i)-\sum_{i=1}^{n b} D B_{i} \Omega \Delta U^{T}(k-i), \\
& \bar{\rho}(k)=\bar{\Theta}_{G}^{T}(k-1) \Phi\left(k, \widehat{\Theta}_{G}\right) \\
&-\sum_{i=1}^{n b} B_{i} \Omega U(k-i) \\
&+\sum_{i=1}^{n b} B_{i} \widehat{\Omega} U^{T}(k-i) \\
&+\sum_{i=1}^{n b} \widehat{D} \widehat{B_{i}} \widehat{\Omega} \Delta U^{T}(k-i) \\
&-\sum_{i=1}^{n b} D B_{i} \widehat{\Omega} \Delta U^{T}(k-i) .
\end{aligned}
$$

So

$$
\bar{\rho}(k)=\bar{Y}(k)+\xi(k)
$$

where

$$
\begin{aligned}
& \xi(k)= \sum_{i=1}^{n b} B_{i}(\widehat{\Omega}-\Omega) U^{T}(k-i) \\
&+\sum_{i=1}^{n b}\left(\widehat{D} \widehat{B}_{i}-D B_{i}\right) \widehat{\Omega} \Delta U^{T}(k-i), \\
& \bar{Y}(k)=\bar{\Theta}_{G}^{T}(k-1) \Phi\left(k, \widehat{\Theta}_{G}\right) .
\end{aligned}
$$

\section{Acknowledgment}

This work was supported by the Ministry of the Higher Education and Scientific Research in Tunisia.

\section{References}

[1] V. B. Kolmanovskii, S.-I. Niculescu, and K. Gu, "Delay effects on stability: a survey," in Proceedings of the 38th IEEE Conference on Decision and Control (CDC '99), vol. 2, pp. 1993-1998, December 1999.

[2] J.-P. Richard, "Time-delay systems: an overview of some recent advances and open problems," Automatica, vol. 39, no. 10, pp. 1667-1694, 2003.

[3] A. Elnaggar, G. A. Dumont, and A.-L. Elshafei, "New method for delay estimation," in Proceedings of the 29th IEEE Conference on Decision and Control, vol. 3, pp. 1629-1630, December 1990.

[4] E. Hidayat and A. Medvedev, "Laguerre domain identification of continuous linear time delay systems from impulse response data," in Proceedings of the 18th International Federation of Automatic Control World Congress, 2011.

[5] M. de la Sen, "Robust adaptive control of linear time-delay systems with point time-varying delays via multiestimation," Applied Mathematical Modelling, vol. 33, no. 2, pp. 959-977, 2009.
[6] O. Gomez, Y. Orlov, and I. V. Kolmanovsky, "On-line identification of SISO linear time-invariant delay systems from output measurements," Automatica, vol. 43, no. 12, pp. 2060-2069, 2007.

[7] Q.-G. Wang and Y. Zhang, "Robust identification of continuous systems with dead-time from step responses," Automatica, vol. 37, no. 3, pp. 377-390, 2001.

[8] S. Ahmed, B. Huang, and S. L. Shah, "Parameter and delay estimation of continuous-time models using a linear filter," Journal of Process Control, vol. 16, no. 4, pp. 323-331, 2006.

[9] S. V. Drakunov, W. Perruquetti, J.-P. Richard, and L. Belkoura, "Delay identification in time-delay systems using variable structure observers," Annual Reviews in Control, vol. 30, no. 2, pp. 143-158, 2006.

[10] X. M. Ren, A. B. Rad, P. T. Chan, and W. L. Lo, "Online identification of continuous-time systems with unknown time delay," IEEE Transactions on Automatic Control, vol. 50, no. 9, pp. 1418-1422, 2005.

[11] W. Gao, M.-L. Zhou, Y.-C. Li, and T. Zhang, "An adaptive generalized predictive control of time-varying delay system," in Proceedings of the 2nd International Conference on Machine Learning and Cybernetics, pp. 878-881, Shanghai, China, August 2004.

[12] Y. Orlov, L. Belkoura, J. P. Richard, and M. Dambrine, "Adaptive identification of linear time-delay systems," International Journal of Robust and Nonlinear Control, vol. 13, no. 9, pp. 857-872, 2003.

[13] L. Ettaleb, G. A. Dumont, and M. S. Davies, "Extended offline least-squares method for parameter identification and time delay estimation," in Proceedings of the 37th IEEE Conference on Decision and Control (CDC '98), pp. 3423-3428, December 1998.

[14] S. Bedoui, M. Ltaief, and K. Abderrahim, "New results on discrete time delay systems," International Journal of Automation and Computing, vol. 9, no. 6, pp. 570-577, 2012.

[15] S. Bedoui, M. Ltaief, and K. Abderrahim, "Nonlinear approach for the identification of discrete time delay systems," in Proceedings of the 20th Mediterranean Conference on Control and Automation (MED '12), Barcelona, Spain, 2012.

[16] A. O. Dwyer, "A survey of techniques for the estimation and compensation of processes with time delay," Tech. Rep., 2000.

[17] P. J. Gawthrop and M. T. Nihtilä, "Identification of time delays using a polynomial identification method," Systems \& Control Letters, vol. 5, no. 4, pp. 267-271, 1985.

[18] S. W. S. Su Whan Sung and I.-B. Lee, "Prediction error identification method for continuous-time processes with time delay," Industrial and Engineering Chemistry Research, vol. 40, no. 24, pp. 5743-5751, 2001.

[19] T. Zhang and Y.-C. Li, "A fuzzy smith control of time-varying delay systems based on time delay identification," in Proceedings of the International Conference on Machine Learning and Cybernetics, vol. 1, pp. 614-619, November 2003.

[20] W. Gao, Y. C. Li, G. J. Liu, and T. Zhang, "An adaptive fuzzy smith control of time-varying processes with dominant and variable delay," in Proceedings of the American Control Conference, vol. 1, pp. 220-224, June 2003.

[21] W. X. Zheng and C. B. Feng, "Identification of stochastic time lag systems in the presence of colored noise," Automatica, vol. 26, no. 4, pp. 769-779, 1990.

[22] Y. Orlov, L. Belkoura, M. Dambrine, and J. P. Richard, "On identifiability of linear time-delay systems," IEEE Transactions on Automatic Control, vol. 47, no. 8, pp. 1319-1324, 2002. 
[23] S. Bedoui, M. Ltaief, and K. Abderrahim, "A new recursive algorithm for simultaneous identification of discrete time delay systems," in Proceedings of the 16th IFAC Symposium on System Identification (SYSID '12), Brussel, Belgium, 2012.

[24] S. Bedoui, M. Ltaief, and K. Abderrahim, "Using a recursive least square algorithm for identification of interconnected linear discretetime delay multivariable systems," in Proceedings of the 17th IEEE International Conference on Methods \& Models in Automation \& Robotics (MMAR '12), Miedzyzdroje, Poland, 2012.

[25] S. Bedoui, M. Ltaief, and K. Abderrahim, "New method for the systematic determination of the model's base of time varying delay system," International Journal of Computer Applications, vol. 46, no. 1, 2012.

[26] J. Roe, R. Gao, and A. O’Dwyer, "Identification of a timedelayed process model using an overparameterisation method," in Proceedings of the China-Ireland International Conference on Information and Communications Technologies (CIICT '07), August 2007.

[27] S. Selvanathan and A. K. Tangirala, "Time-delay estimation in multivariate systems using Hilbert transform relation and partial coherence functions," Chemical Engineering Science, vol. 65, no. 2, pp. 660-674, 2010.

[28] B. Ni, D. Xiao, and S. L. Shah, "Time delay estimation for MIMO dynamical systems-with time-frequency domain analysis," Journal of Process Control, vol. 20, no. 1, pp. 83-94, 2010.

[29] T. S. Söderström and P. Stoica, System Identification, Prentice Hall International Series in Systems and Contol Engineering, 1989.

[30] Y. Xiao, F. Ding, Y. Zhou, M. Li, and J. Dai, “On consistency of recursive least squares identification algorithms for controlled auto-regression models," Applied Mathematical Modelling, vol. 32, no. 11, pp. 2207-2215, 2008.

[31] O. Nelles, Nonlinear System Identification: From Classical Approach to Neural Networks and Fuzzy Models, Springer, Berlin, Germany, 2001.

[32] L. Mišković, A. Karimi, D. Bonvin, and M. Gevers, "Correlation-based tuning of decoupling multivariable controllers," Automatica, vol. 43, no. 9, pp. 1481-1494, 2007.

[33] B. de Moor, P. de Gersem, B. de Schutter, and W. Favoreel, "Daisy: a database for identification of systems," Journal A, vol. 38, no. 3, pp. 4-5, 1997.

[34] L. Lapidus and G. F. Pinder, Numerical Solution of Partial Differential Equations in Science and Engineering, John Wiley \& Sons, New York, NY, USA, 1982. 


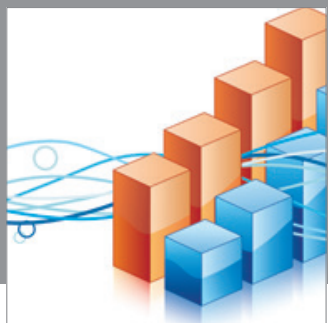

Advances in

Operations Research

mansans

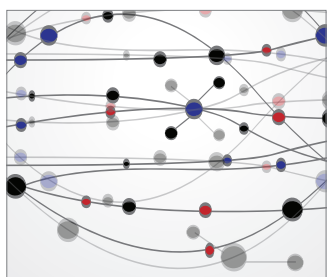

The Scientific World Journal
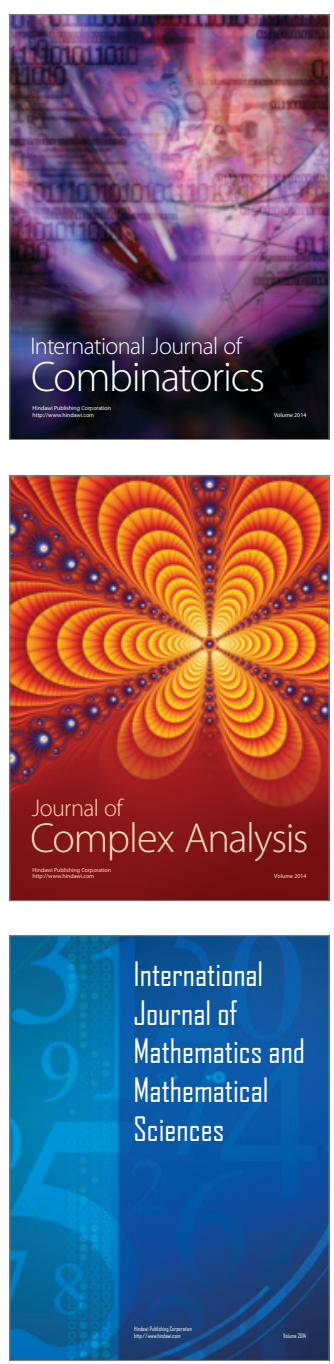
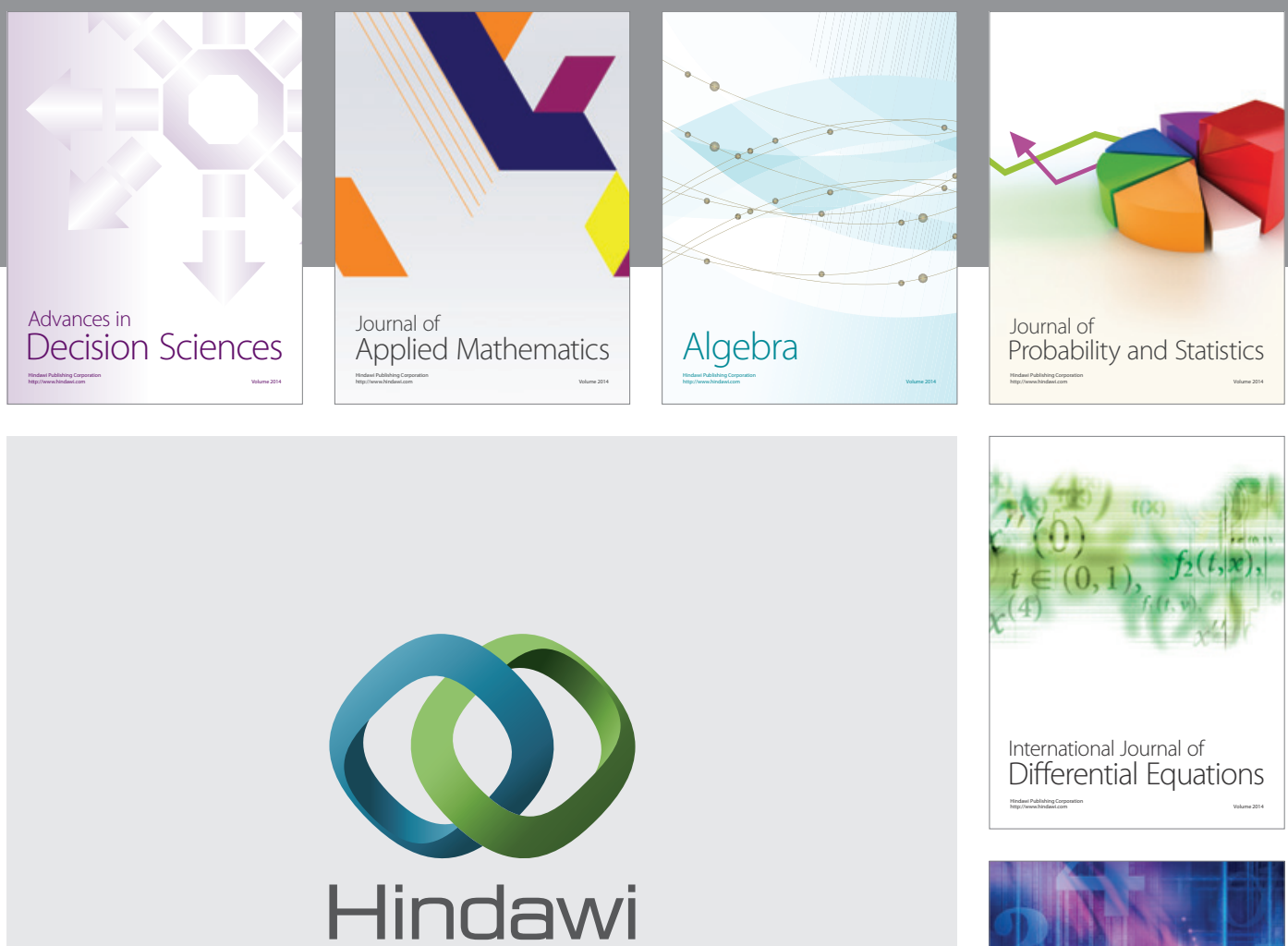

Submit your manuscripts at http://www.hindawi.com
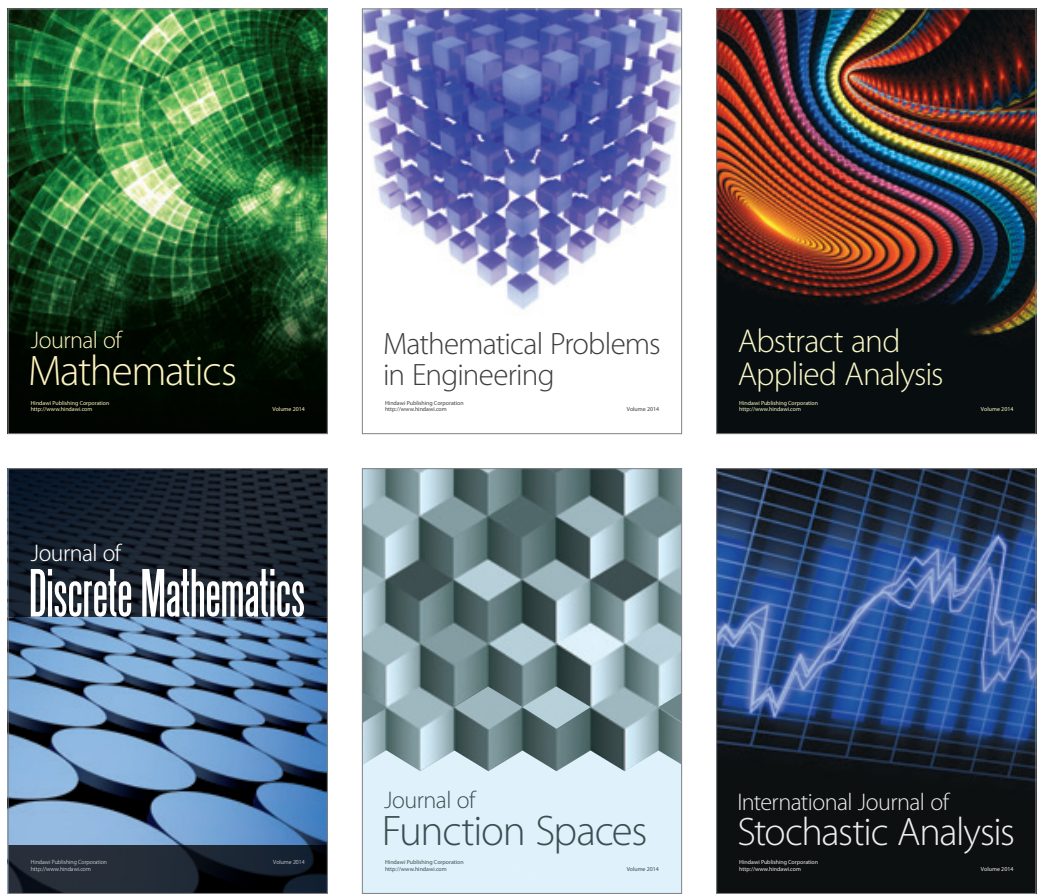

Journal of

Function Spaces

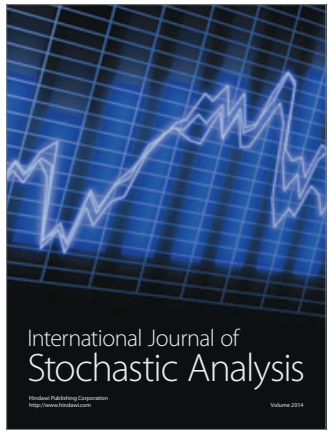

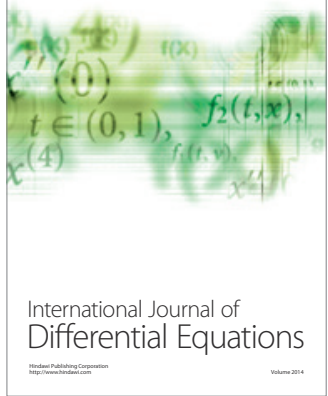
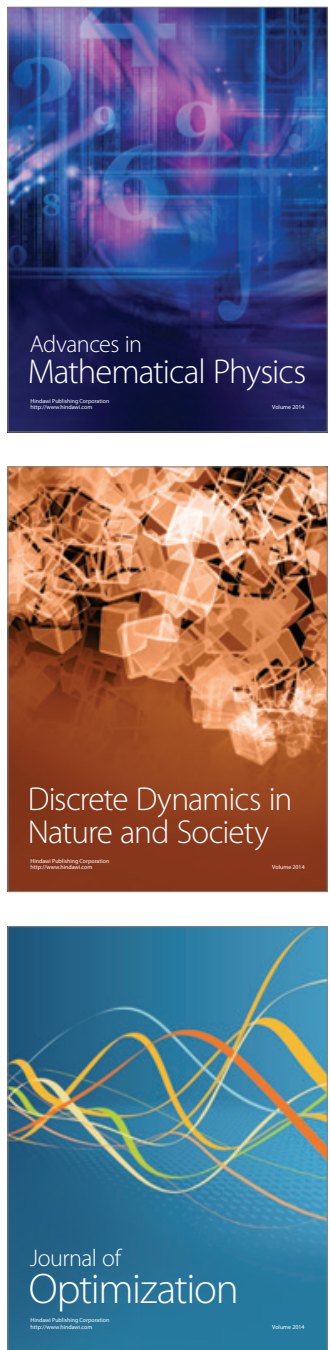